\title{
Tumor-specific interendothelial adhesion mediated by FLRT2 facilitates cancer aggressiveness
}

\author{
Tomofumi Ando, ${ }^{1,2}$ Ikue Tai-Nagara, ${ }^{1}$ Yuki Sugiura, ${ }^{3}$ Dai Kusumoto, ${ }^{4}$ Koji Okabayashi, ${ }^{2}$ Yasuaki Kido, ${ }^{1}$ Kohji Sato, ${ }^{5}$ Hideyuki Saya, \\ Sutip Navankasattusas, ${ }^{7}$ Dean Y. Li, ${ }^{7,8,9,10,11,12,13}$ Makoto Suematsu, ${ }^{3}$ Yuko Kitagawa, ${ }^{2}$ Elena Seiradake, ${ }^{14}$ Satoru Yamagishi, ${ }^{5}$ and \\ Yoshiaki Kubota' \\ DDepartment of Anatomy, ${ }^{2}$ Department of Surgery, ${ }^{3}$ Department of Biochemistry, and ${ }^{4}$ Department of Cardiology, Keio University School of Medicine, Tokyo, Japan. ${ }^{5}$ Department of Organ \& Tissue Anatomy, \\ Hamamatsu University School of Medicine, Hamamatsu, Japan. ${ }^{6}$ Division of Gene Regulation, Institute for Advanced Medical Research, Keio University School of Medicine, Tokyo, Japan. ${ }^{7}$ Department of \\ Medicine, Program in Molecular Medicine, ${ }^{8}$ Department of Oncological Sciences, ${ }^{9}$ Department of Human Cenetics, ${ }^{10}$ ARUP Laboratories, ${ }^{11}$ Division of Cardiovascular Medicine, Department of Medicine, and \\ ${ }^{12}$ Department of Cardiology, VA Salt Lake City Health Care System, University of Utah, Salt Lake City, Utah, USA. ${ }^{3}$ Key Laboratory for Human Disease Gene Study, Sichuan Academy of Medical Sciences and \\ Sichuan Provincial People's Hospital, Chengdu, China. ${ }^{14}$ Department of Biochemistry, University of Oxford, Oxford, United Kingdom.
}

\begin{abstract}
Blood vessel abnormalization alters cancer cell metabolism and promotes cancer dissemination and metastasis. However, the biological features of the abnormalized blood vessels that facilitate cancer progression and whether they can be targeted therapeutically have not been fully investigated. Here, we found that an axon guidance molecule, fibronectin leucine-rich transmembrane protein 2 (FLRT2), is expressed preferentially in abnormalized vessels of advanced colorectal cancers in humans and that its expression correlates negatively with long-term survival. Endothelial cell-specific deletion of Flrt2 in mice selectively pruned abnormalized vessels, resulting in a unique metabolic state termed "oxygen-glucose uncoupling," which suppressed tumor metastasis. Moreover, Flrt2 deletion caused an increase in the number of mature vessels, resulting in a significant increase in the antitumor effects of immune checkpoint blockers. Mechanistically, we found that FLRT2 forms noncanonical interendothelial adhesions that safeguard against oxidative stress through homophilic binding. Together, our results demonstrated the existence of tumor-specific interendothelial adhesions that enable abnormalized vessels to facilitate cancer aggressiveness. Targeting this type of adhesion complex could be a safe and effective therapeutic option to suppress cancer progression.
\end{abstract}

\section{Introduction}

Development and maintenance of the body's organs require an adequate blood supply to bring oxygen and nutrients to the tissues (1). Tumor growth also depends on formation of new blood vessels from existing ones. However, hastily built new vessels tend to be leaky and functionally immature; these are referred to as "abnormalized" (2). Impaired perfusion and oxygenation caused by vessel abnormalization activates a metabolic switch in cancer cells and promotes focal invasion and distant metastasis (2).

VEGF is the most potent angiogenic factor that drives tumor angiogenesis. VEGF inhibitors are used widely to treat various types of human cancers (2-4). Although VEGF inhibitors suppress tumor progression, the therapeutic effects are sometimes limited; paradoxically, VEGF inhibitors can promote aggressiveness by increasing tumor hypoxia or the number of abnormalized vessels (5-8). Moreover, damage to normal vessels can cause severe adverse effects, including cerebral hemorrhage and intestinal

Authorship note: SY and YK contributed equally to this work.

Conflict of interest: The authors have declared that no conflict of interest exists. Copyright: ( 2022 , Ando et al. This is an open access article published under the terms of the Creative Commons Attribution 4.0 International License.

Submitted: July 27, 2021; Accepted: January 28, 2022; Published: March 15, 2022

Reference information: J Clin Invest. 2022;132(6):e153626.

https://doi.org/10.1172/JCl153626. perforation $(9,10)$. In light of these clinical situations, it will be important to identify drugs that target abnormalized but not normal blood vessels, which could be used in combination with immunotherapeutic drugs $(11,12)$ to combat cancer.

Fibronectin leucine-rich transmembrane protein 2 (FLRT2), a member of the FLRT family of proteins, acts as a repulsive ligand of the UNC5 receptor family; it was initially identified as a chemorepellent in neurons (13-15). Outside of the nervous system, FLRT2 is expressed in endothelial cells in the placental labyrinth, and FLRT2 contributes to its proper formation (16). It was also reported that FLRT2 possesses dual functions: heterophilic binding to UNC5B, which causes cellular repulsion, and homophilic binding, which supports intercellular adhesion (15). FLRT proteins also interact with adhesion GPCRs of the latrophilin family (17), with established functions in brain development.

Interendothelial junctions, which are essential for vascular integrity and function, comprise tight junctions, adherens junctions, and a variety of other adhesion molecules such as PECAM1 and angiopoietins (18). These junctions maintain a stable vascular system and control vascular permeability. In tumors, loss of interendothelial adhesion is a hallmark of abnormalized vessels, which promote cancer progression by enabling transvascular migration of tumor cells $(2,18,19)$.

Here, we analyzed human colorectal cancer samples and found that FLRT2 is expressed abundantly in endothelial cells 
lining abnormalized vessels. Deletion of endothelial Flrt2 in mice selectively ablated these abnormalized vessels, thereby suppressing tumor invasion and metastasis without triggering the metabolic switch. Mechanistically, Flrt2 facilitates interendothelial adhesion by forming homophilic bonds, thereby protecting cells from oxidative stress. Taken together, these findings suggest that abnormalized vessels exploit noncanonical interendothelial adhesions, which are different from the canonical junctions in healthy vessels, to disseminate tumor cells.

\section{Results}

FLRT2 is expressed in the tumor endothelial cells of advanced human colorectal cancers. Previously, we showed that Flrt2 is expressed by endothelial cells in the placental labyrinth, but not in other vascular beds, during mouse embryogenesis (16). Because the placenta produces ROS and is exposed to oxidative stress (20), we sought to examine expression of FLRT2 in abnormalized tumor vessels, which are also exposed to oxidative stress (21). We examined expression of FLRT2 by immunostaining tissues from a cohort of 47 patients with colorectal cancer who had undergone surgical resection. Colorectal cancers are epithelial cell-derived malignant tumors that infiltrate the submucosal, muscularis, and serosal layers; therefore, we examined expression of FLRT2 in superficial and progressive areas (Figure 1A). Interestingly, FLRT2 was expressed abundantly in $\mathrm{CD}_{3} 4^{+}$endothelial cells within areas of progression in stage IV cancer samples, but far less so in superficial areas (Figure $1, \mathrm{~B}-\mathrm{G})$. Blood vessels in the progressive area were apparently angiogenic, with many structures with an appearance of sprouting (Supplemental Figure 1A; supplemental material available online with this article; https://doi.org/10.1172/JCI153626DS1). In addition, while there was no detectable expression of FLRT2 in normal colon tissues or stage I tumors, strong expression was observed in progressive areas of stage II, III, and IV tumors, which are defined as advanced cancers (Figure 1, H-M, and Supplemental Figure 1B). Because these results suggest that expression of endothelial FLRT2 is related to long-term prognosis, we examined another cohort of 66 patients who underwent curative surgical resection for pathological stage II or III colorectal cancer and were followed up for 5 years (Supplemental Figure 1C). Strikingly, Kaplan-Meier curves for recurrence-free survival (RFS) showed that patients with high expression of endothelial FLRT2 had a significantly poorer prognosis than those with low expression (Figure 1N). Furthermore, multivariate analyses using a Cox proportional hazards model revealed that high expression of FLRT2 by endothelial cells was an independent risk factor for RFS, regardless of tumor stage (Supplemental Figure 1D). Taken together, these data suggest that expression of FLRT2 is an independent biomarker for predicting prognosis of colorectal cancers (in addition to canonical staging). Next, we asked what triggers expression of FLRT2 by endothelial cells. In agreement with a previous report (22), we found that VEGF upregulated expression of FLRT3 (another member of the FLRT family), but not FLRT2, in cultured human umbilical vein endothelial cells (HUVECs; Supplemental Figure 2A). Among other stimuli, we found that elevation of ROS mediated by KU55933, an ATM inhibitor (21), or treatment with $\mathrm{H}_{2} \mathrm{O}_{2}$ significantly increased expression of FLRT2 by HUVECs (Figure 10 and Supplemental Figure 2B). Increased expression of FLRT2 protein was localized to intercellular junctions as well as the cytoplasm (Figure 1, P-U). Flrt2 was also abundantly expressed in aberrantly expanding neovessels, which are known to be exposed to high oxidative stress (21), in ischemic retinopathy but not in surrounding stable vessels (Supplemental Figure 2, C and D). This expression of FLRT2 was suppressed by administration of the antioxidant $\mathrm{N}$-acetyl-L-cysteine (Supplemental Figure 2, C-F). In combination with the clinical data, these data indicate that FLRT2 is expressed by endothelial cells lining tumor vessels that are exposed to high levels of oxidative stress.

Endothelial Flrt2 sustains abnormalized tumor vessels in mice. Next, we used a syngeneic murine B16 melanoma model to evaluate the bona fide role of endothelial FLRT2 in tumor progression. Because conventional and endothelial cell-specific Flrt2-knockout mice are embryonically lethal $(16,23)$, we generated tamoxifen-inducible endothelial cell-specific Flrt2-knockout mice (Cdh5-BAC-Cre ${ }^{E R T 2} F l r t 2^{f l / f l}$ mice, referred to hereafter as Flrt $2^{i \triangle E C}$ mice). We subcutaneously implanted these mice with B16 cells and then injected them with 4-hydroxytamoxifen (4OHT) for 4 consecutive days (Figure 2A). This protocol effectively deleted Flrt2 from the tumor endothelial cells (Figure 2B). At 10 days after implantation, tumor growth was moderately suppressed in Flrt2 ${ }^{i \Delta E C}$ mice (Figure 2, C-E). Histologically, Flrt2 ${ }^{i \triangle E C}$ mice showed a moderate reduction in the total number of tumor vessels and a marked reduction in the area of intratumoral hemorrhage (Figure 2, F-K). Considering intratumor variability of the number of blood vessels (24), we counted all of the vessels in the entire field within the plane of maximum cut of tumor samples. The percentage of blood vessels covered by smooth muscle actin ${ }^{+}\left(\mathrm{SMA}^{+}\right)$ mural cells (25), namely stable vessels, was markedly increased in Flrt $2^{i \triangle E C}$ mice (Figure 2, L, M, and T). Endothelial proliferation in Flrt $2^{i \triangle E C}$ mice was lower than that in control mice (Figure 2, N, $\mathrm{O}$, and U). Tumors in Flrt ${ }^{i \triangle E C}$ mice showed reduced intratumor hypoxia, accompanied by increased vessel perfusion (Figure 2, $\mathrm{P}-\mathrm{S}, \mathrm{V}$, and $\mathrm{W})$. Intracardiac injection of $10 \mathrm{kDa}$ dextran showed decreased vessel leakiness in Flrt $2^{i \triangle E C}$ mice (Supplemental Figure 3, A and B), supporting the reduced hemorrhaging data. Accumulation of macrophages in the hemorrhagic lesion was not apparent (Supplemental Figure 3, C and D). Healthy vessels outside the tumor, postnatal retinal vascularization, and wound healing were not affected by Flrt2 deletion (Supplemental Figure 4, A-H). Similar to the transient effect of VEGF blockade during the "normalization window" $(26,27)$, deletion of endothelial Flrt2 increased the tumor-suppressing effects of a cytotoxic agent, cisplatin (28, 29), and those of immunotherapeutic drugs (anti-PD1 antibodies) (Figure 3). Next, we injected tamoxifen 14 days after transplantation and examined the immediate effect of intermittent FLRT2 deletion, which revealed ablation of abnormalized vessels and a reduction in hypoxia in Flrt $2^{i \triangle E C}$ mice as in the primary protocol for tamoxifen (Supplemental Figure 5). These data suggest that FLRT2 indeed functions in vessel maintenance.

Deletion of endothelial Flrt2 induces oxygen-glucose uncoupling in tumors. Next, we conducted a metabolomic analysis to better understand how tumors respond to deletion of endothelial Flrt2. Reprogramming of glucose metabolism in cancer cells accelerates not only aerobic glycolysis but also anabolic pathways that generate macromolecules (e.g., nucleotides) via the pentose phosphate 


Figure 1. Endothelial expression of FLRT2 in advanced human colorectal cancers. (A) Schematic diagram depicting the location of the progressive and superficial areas in human colorectal cancer. (B-C) Immunohistochemical analysis of FLRT2 and CD34 expression in serial sections of paraffin-embedded samples cut from resected tumors from stage IV cases. Endothelial cells in the progressive area (closed arrowheads), but not those in the superficial area (open arrowheads), express FLRT2. (H-K) Immunohistochemical analysis of FLRT2 expression in the progressive area of tumors from individuals with various stages of colorectal cancer. Endothelial cells in tumors at advanced stages show FLRT2 expression (arrowheads). (L) Quantification of FLRT2 expression, as measured by the IHC score ( $n=46$ each). (M) Quantification of FLRT2 expression in the progressive area of tumors at various stages ( $n=13$ [stage I], 23 [stage II/III], 10 [stage IV]). The red line represents regression. (N) Kaplan-Meier curve showing recurrence-free survival of patients with stage II or III colorectal cancer, stratified according to FLRT2 expression (high- and low-scoring tumors). The log-rank test was used to compare differences between groups $(n=66)$. (0) Relative expression of FLRT2 and FLRT3 under various culture conditions ( $n=4$ each). (P-U) Immunocytochemistry analysis of HUVECs. Localization of FLRT2 proteins at the intracellular junctions (arrowheads) increased markedly after oxidative stress induced by KU55933. Representative images for 3 independent experiments are shown. Scale bars: $500 \mu \mathrm{m}$ (B and $\mathbf{C}) ; 50 \mu \mathrm{m}$ (D-K and $\mathbf{P}-\mathbf{U}$ ). Data are presented as the mean \pm SD. Comparisons between mean values of 2 groups were evaluated using a 2-tailed Student's $t$ test. Comparisons among multiple groups ( $\mathbf{M}$ and $\mathbf{0}$ ) were evaluated using 2-way ANOVA followed by Bonferroni's multiple-comparison test. Kaplan-Meier curves and the log-rank test were used to compare survival among the groups.

pathway (PPP) and glycogens synthesis via the uridine diphosphate/glucose pathway, thereby driving tumor progression (30). Moreover, vessel leakiness is supposed to affect glucose uptake of tumor cells, particularly in highly glycolytic tumors such as B16 melanoma. Therefore, we injected mice intraperitoneally with full-labeled glucose ([U-133 $\left.\mathrm{C}_{6}\right]$ glucose) and traced the metabolic fate of ${ }^{13} \mathrm{C}_{6}$ glucose-derived carbon atoms. Volcano plot analysis shows that the flow toward the PPP was reduced significantly in Flrt ${ }^{i \triangle E C}$ mice (Figure 4, A and B, and Supplemental Figure 6); the ratio of the reduction was greater than that for glycolysis (Figure $4 \mathrm{C}$ ) and for the TCA cycle (Figure 4D). Imaging mass spectrometry (IMS) revealed reductions in key metabolites, namely lactate (a glycolytic end product) and inosine monophosphate (an intermediate in the PPP) (Figure 4, E-L). To evaluate glucose utilization by tumor cells, we injected mice intraperitoneally with 2-deoxy$\mathrm{D}$-glucose (2-DG), which is taken up by cells and converted into a phosphorylated form (2DG-6-phosphate [2DG-6P]) that cannot undergo further downstream glycolysis (31). The amount of 2DG-6P in the tissues of Flrt ${ }^{\mathrm{i} A E C}$ mice was reduced significantly (Figure 4, M and N, and Supplemental Figure 7). Moreover, when we checked the distribution of 2,3-DPG, a metabolite that is enriched in erythrocytes (32), we found that most of the 2DG-6P was localized in the 2,3-DPG ${ }^{+}$hemorrhagic area (Figure 4, O-Q), suggesting that the injected 2-DG leaked out of abnormalized vessels in control mice but not in Flrt $2^{i \triangle E C}$ mice. These data indicate that the reduction in abnormalized vessels in Flrt ${ }^{i \triangle E C}$ mice increased oxygen provision and decreased glucose leakage and that this oxygen-glucose uncoupling contributes to the suppression of not only aerobic glycolysis, but also anabolic pathways.
Deletion of Flrt2 prevents tumor metastases and spontaneous tumor formation. The data above indicate that the deletion of endothelial Flrt2 efficiently suppresses tumor angiogenesis without triggering metabolic reprogramming. Thus, we utilized a syngeneic mouse cancer model based on AXT cells, a highly metastatic osteosarcoma cell line (33). Growth of primary tumors in the back skin of Flrt $2^{i \mathrm{iEC}}$ mice was slightly inhibited (Figure 5, A and B). Kaplan-Meier curves for overall survival showed that the 35 day overall survival was significantly higher for Flrt $2^{i \Delta E C}$ mice than for control mice (Figure 5C). Tumors in Flrt $2^{i \Delta E C}$ mice showed reduced intratumor hypoxia (Supplemental Figure 8A). Accordingly, intravasation of tumor cells derived from primary tumors and resultant metastasis to the liver and lungs were markedly lower in Flrt $2^{i A E C}$ mice (Figure 5, D-L, and Supplemental Figure 8B). To test the contribution of Flrt2 in the premetastatic niche, we examined the expression of Flrt2 in endothelial cells before and after transplantation of AXT cells. We did not observe any apparent changes in the expression of Flrt2 (Supplemental Figure 8C). The metastasis phenotype of Flrt $2^{i \Delta E C}$ mice was in stark contrast to that of endothelial Vegfr2-knockout mice (Cdh5-BAC-Cre ${ }^{E R T 2} V e g f r-$ $2^{f / f l}$, referred to hereafter as $\mathrm{Veg} f \mathrm{r}^{\mathrm{i}}{ }^{\mathrm{NEC}}$ mice); AXT cells transplanted into Vegfr $2^{i \Delta E C}$ mice showed increased metastasis to the liver, despite the decreased growth of primary tumors, likely due to the increase in hypoxia caused by excessive destruction of tumor vessels (Supplemental Figure 9). The expression of endothelial Flrt2 in B16 tumors transplanted into Vegfri ${ }^{i \triangle E C}$ mice was not impaired (Supplemental Figure 10), in agreement with the in vitro data showing that VEGF signaling is not related to the expression of FLRT2 (Supplemental Figure 2A). From the viewpoint of actual human cancers, there is a need to study more clinically relevant models that recapitulate modes of cancer progression, such as spontaneous tumorigenesis $(34,35)$. Therefore, we utilized APC${ }_{\mathrm{Min} /}$ mice (36). At 18 weeks of age, $A P C^{\mathrm{Min} /}+\mathrm{Flrt} 2^{i \Delta E C}$ mice developed fewer tumors in both the colon and small intestine than $A P C^{\mathrm{Min} /+}$ mice (Figure $5, \mathrm{M}-\mathrm{T}$ ). The number of tumors greater than $3 \mathrm{~mm}$ in diameter in the small intestine of $A P C^{\mathrm{Min} /} \mathrm{Flrt} 2^{\mathrm{i} E \mathrm{CC}}$ mice was significantly lower than that in $A P C^{\mathrm{Min} / \mathrm{H}}$ mice (Figure 5, Q and S).

Intercellular homophilic binding of FLRT2 protects ECs from oxidative stress. Because Flrt2 acts primarily as a repulsive ligand for the Unc5b receptor $(14,16)$, we injected endothelial cellspecific Unc5b-knockout mice (Cdh5-BAC-Cre ${ }^{E R T 2} U n c 5 b^{f / f}$; referred to hereafter as $U n c 5 b^{i \triangle E C}$ mice) with B16 melanoma cells. Unexpectedly, tumor growth, vascularization, and intratumor hemorrhage were not altered significantly in $U n c 5 b^{i \Delta E C}$ mice (Supplemental Figure 11, A-E). Therefore, to explore alternative mechanisms underlying the vascular normalizing effect of Flrt2 deletion, we performed comparative transcriptomics analyses using HUVECs subjected to si-RNA-mediated knockdown of FLRT2. Principal components analyses and Pearson correlation matrix analysis of RNA-Seq data revealed global changes in the transcriptome of HUVECs treated with the si-Control or si-FLRT2 (Figure 6, A-D). Extraction of differentially expressed genes (DEGs) and gene set enrichment analysis (GSEA) indicated that the genes most affected by si-FLRT 2 were associated with immune responses represented by type I interferon signaling, responses to viruses, and peptide antigen binding (Supplemental Figure 12A and Supplemental Table 1), none of which are 
A

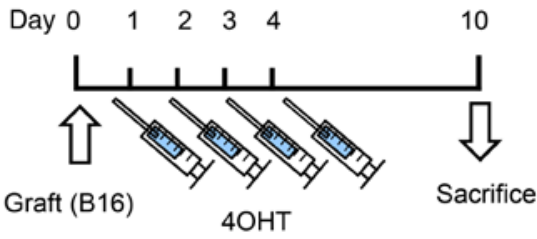

B Cdh5-CreERT2+ CAG-LSL-GFP+
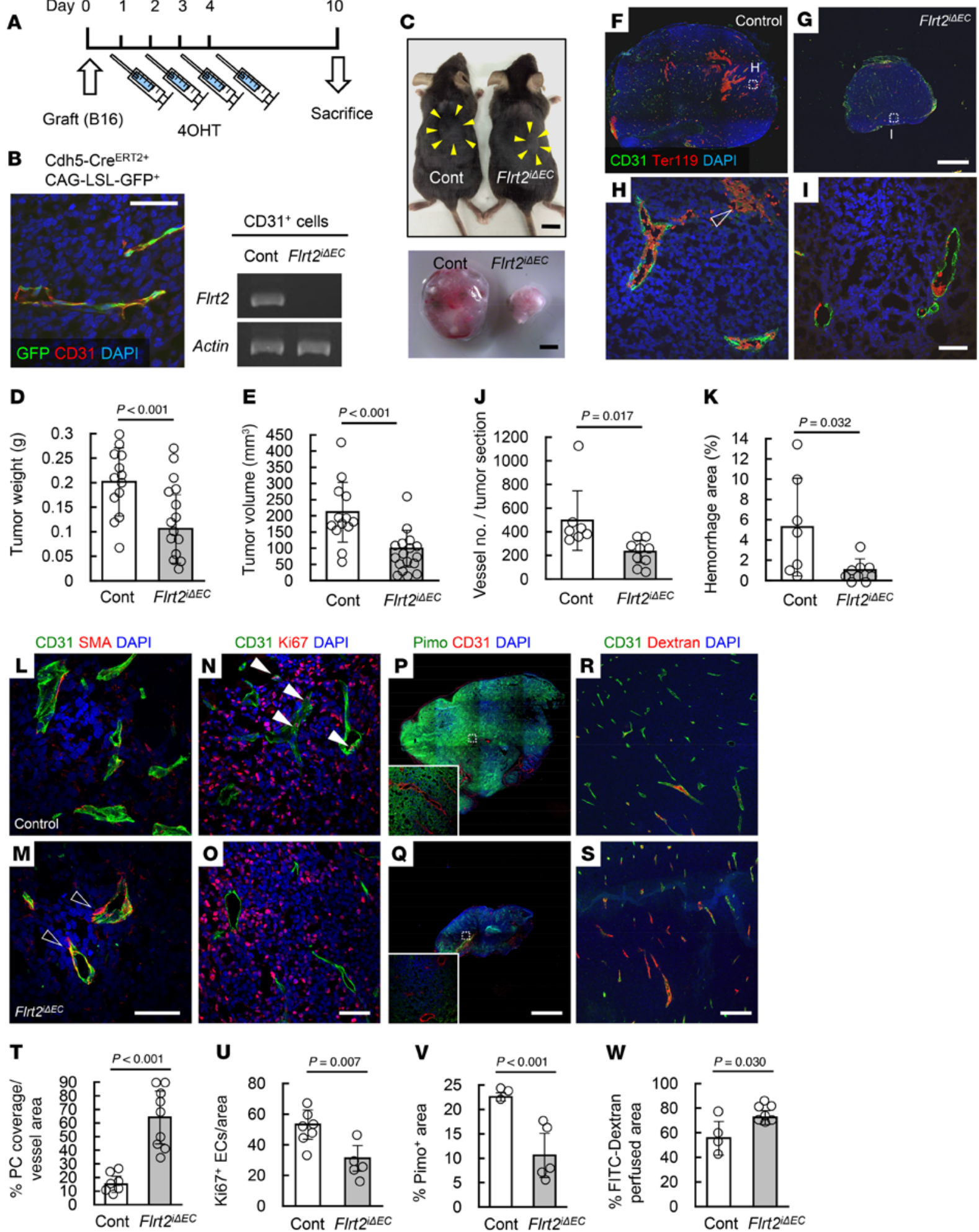

Figure 2. Endothelial FIrt2 sustains abnormalized tumor vessels in mice. (A) Protocol for $40 \mathrm{HT}$ injections and tumor inoculations. (B) Representative IHC of B16 tumors on day $10(n=3)$ and representative PCR results $(n=3)$ showing expression of Flrt2 in isolated CD31+ tumor endothelial cells. (C) Tumors resected from mice 10 days after transplantation of B16 cells into the back skin (arrowheads). (D and E) Measurement of tumor weight and volume ( $n=13$ [control], 15 [Flrt2 ${ }^{i \Delta E c]}$ ). (F-K) Immunohistochemical analysis of tumor sections ( $n=7$ [control], 9 [Flrt2 ${ }^{\text {isEc] }}$ ). Intratumoral hemorrhage (open arrowheads) in tumors from control mice is greater than that in Flrt2 ${ }^{i L E C}$ mice. (L-W) Immunohistochemical analysis of tumor sections ( $n=7,9,7$, and 5 [control], 3, 5, 4, and 7 [Flrt2 $\left.{ }^{\mathrm{i} E C}\right]$ ). Tumors from Flrt2 ${ }^{i E E C}$ mice show reduced endothelial proliferation (closed arrowheads) and increased pericyte coverage (open arrowheads). Scale bars: $1 \mathrm{~cm}$ (C [top]); 2 mm (C [bottom], F, G, P, and Q); $200 \mu \mathrm{m}$ (R and S); $50 \mu \mathrm{m}$ (B, H, I, and L-0). Data are presented as the mean \pm SD. Comparisons between mean values of 2 groups were evaluated using a 2-tailed Student's $t$ test. 
A
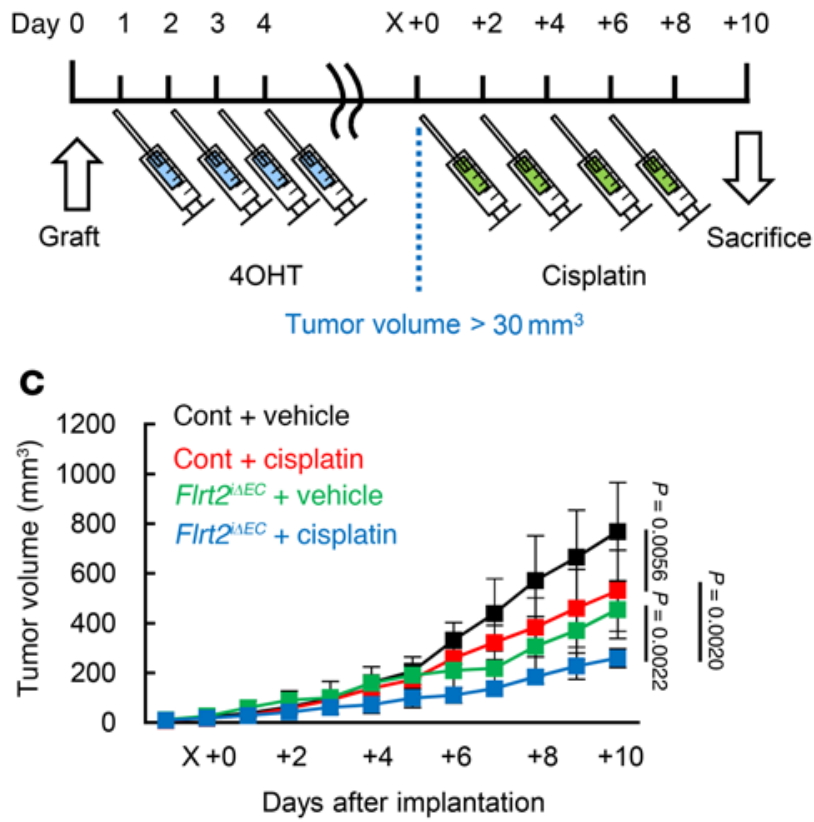

B

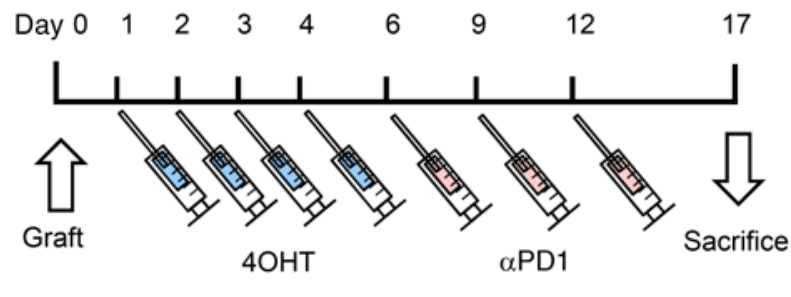

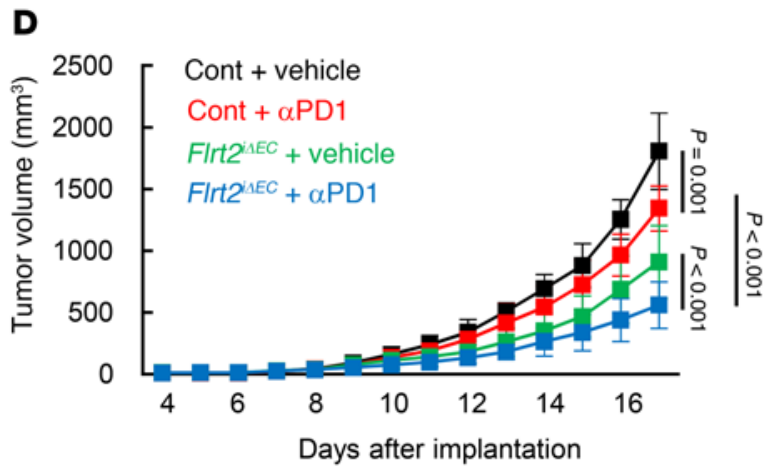

Figure 3. Endothelial Flrt2 deletion potentiates tumor-suppressing effects of cytotoxic and immunotherapeutic drugs. (A) Protocol for injection of $40 \mathrm{OHT}$ and cisplatin treatment $(2.5 \mathrm{mg} / \mathrm{kg}$ ) and for tumor inoculation. (B) Protocol for injection of 40HT, anti-PD1 antibody treatment, and tumor inoculation. (C) Measurement of tumor volume ( $n=14$ [control+vehicle], 11 [control+cisplatin], 8 [Flrt2 ${ }^{\text {isEc }}+$ vehicle], 10 [Flrt2 $2^{\text {isEc }}+$ cisplatin]). (D) Measurement of tumor

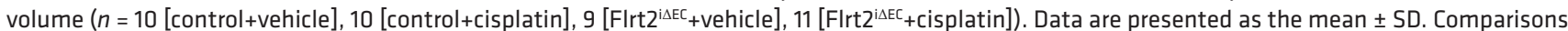
among multiple groups were evaluated using 2-way ANOVA followed by Bonferroni's multiple-comparison test.

relevant to angiogenesis. Therefore, we checked subleading DEGs and ontologies and found that si-FLRT2 downregulated genes related to the cytoskeleton ( $R H O B, R A S A 1, R O C K 1$, etc.) and cell cycle (CDC45, E2F8, MCM1O, etc.), whereas upregulated genes were related to oxidative stress (PID1, ICAM1, PTX3, etc.) and intercellular adhesion (IDO1, CECAM1, LGALS9, etc,) (Figure $6, \mathrm{C}$ and $\left.\mathrm{E}^{-} \mathrm{G}\right)$. We next examined the expression of UNC5 receptors by checking their absolute mRNA amounts (transcripts per kilobase million) in HUVECs. Although the UNC5A, -C, and -D expression was nearly undetectable, UNC5B expression was abundant and was significantly increased by treatment with si-FLRT2 (Supplemental Figure 12B). Considering that FLRT2 can support intercellular adhesion through homophilic binding (Figure 7A) and accumulate at cell-to-cell junctions under the control of ROS (Figure 1, P-U), we suspected that homophilic FLRT2 binding acts adhesively, particularly when ROS are elevated. Treatment of HUVECs with si-FLRT2 alone led to a significant reduction in the number of adherens junctions (Figure 7, B, C, F, G, J, K, and N). This effect of si-FLRT2 was more prominent when intracellular ROS were elevated by KU55933 (Figure 7, B-N). To evaluate the homophilic interaction between FLRT2 molecules more closely, we utilized a synthetic nonhomophilic-binding FLRT2 mutant (FLRT2 ${ }^{\mathrm{R} 186 \mathrm{~N}+\mathrm{D} 188 \mathrm{~T}}$; referred to hereafter as FLRT2 $\left.{ }^{\triangle \mathrm{FF}}\right)$, created by introducing obstacle N-linked glycosylation sites (15). Although wild-type FLRT2 proteins promoted adhesion of HUVECs, FLRT2 ${ }^{\mathrm{AFF}}$ proteins did not (Figure 7O). FLRT2 $2^{\triangle F F}$ proteins also lacked binding to latrophilins, G protein-coupled receptors, suggesting a potential role for latrophilins in the adhesive activity of FLRT2 (37). However, adhesion to wild-type FLRT2 proteins in HUVECs was abolished by knocking down endogenous FLRT2, suggesting that this activity depends on homophilic binding of FLRT2 molecules (Figure 7O). Taken together, these data suggest that homophilic binding of FLRT2 supports interendothelial adhesion.

Homophilic binding of FLRT2 constitutes tumor-specific interendothelial adhesion. The above in vitro data indicate that homophilic binding of Flrt2 protects tumor vessels, which are exposed to high levels of ROS (21). Indeed, we detected Flrt2 proteins in the interendothelial cell junctions of tumors but not in normal skin vessels (Figure 8A). Next, we generated knockin mice lacking homophilic binding of Flrt2 (Flrt2 $2^{\text {R186N+D188T }}$ mice) (Figure 8B). Homozygous mutant mice lacking homophilic Flrt2 (Flrt2 ${ }^{\text {R186N+D188T/R186N+D188T }}$ ) died before birth. Mice lacking homophilic Flrt2 binding specifically in endothelial cells ( $C d h 5-C r e^{E R T 2+} F l r t 2^{f l o x / R 186 N+D 188 T}$; referred to hereafter as $F l r t 2^{i \triangle E C / \triangle F F}$ mice) showed suppression of tumor growth, angiogenesis, and hemorrhaging, similar to those in Flrt $2^{i \Delta E C}$ mice (Figure 8, C-E and N-Q). It should be noted that the FLRT2-latrophilins interaction $(17,37,38)$ could account for this phenotype, in addition to its homophilic binding. A metastatic model based on AXT cells also showed that tumors in Flrt $2^{i \triangle E C / \triangle F F}$ mice demonstrated markedly lower levels of intravasation and metastasis than those in control mice (Figure 8, F-M, R, and S), suggesting that homophilic binding of Flrt2 provides the interendothelial adhesion required for maintenance of abnormalized vessels.

\section{Discussion}

Here, we show that noncanonical interendothelial adhesion mediated by FLRT2 supports rapidly growing abnormalized 
A
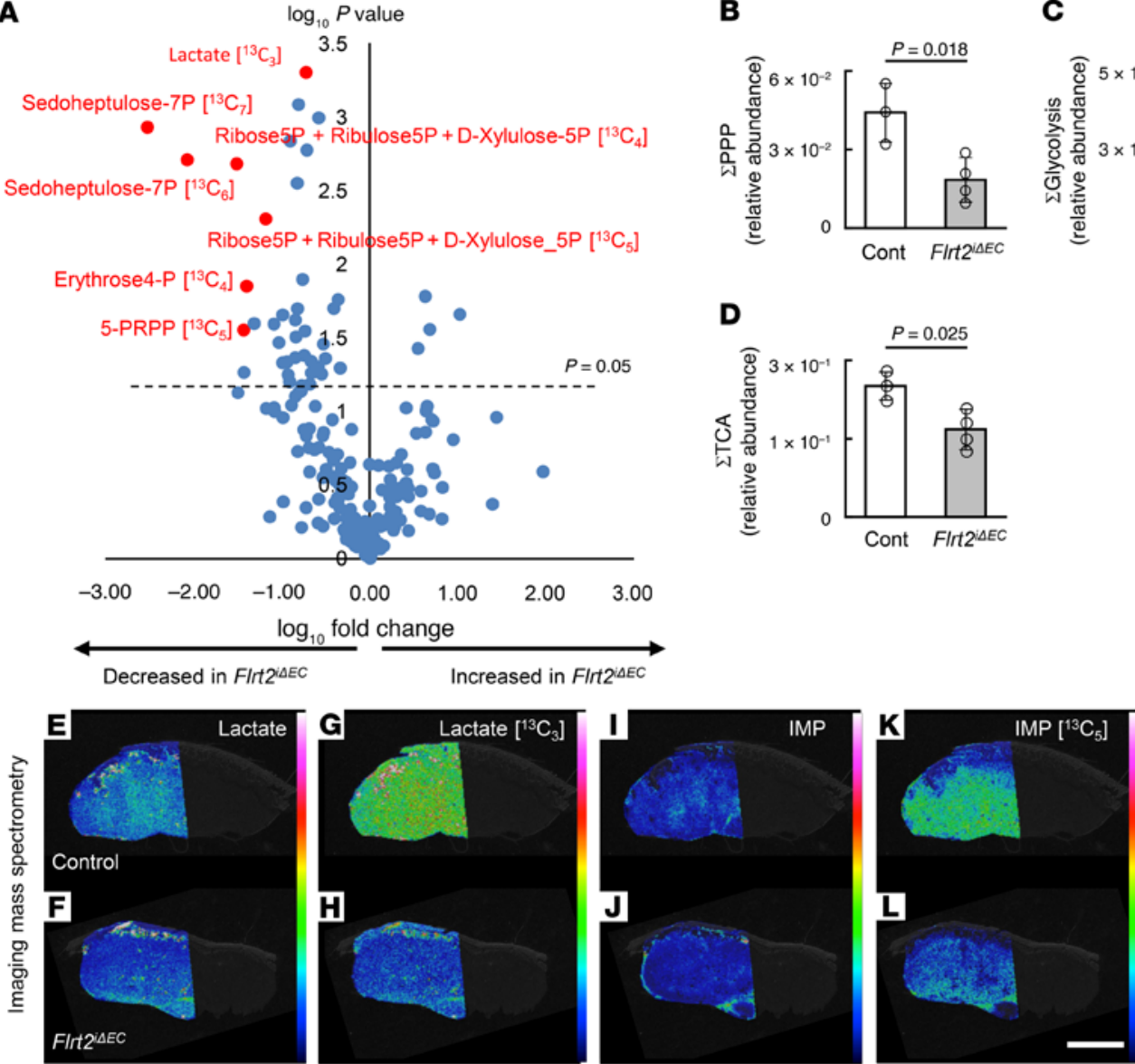

M
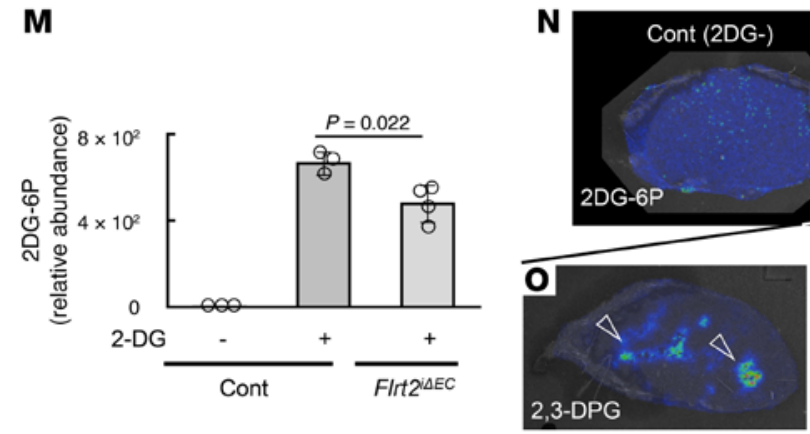

C
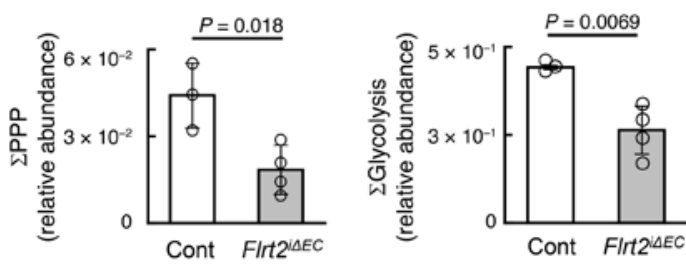

D

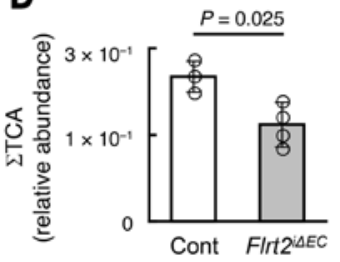

Figure 4. Deletion of endothelial Flrt2 induces oxygen-glucose uncoupling in tumors. (A) Volcano plot showing the amount of metabolites in Flrt $2^{i \Delta E C}$ mice relative to that in control mice. The red dots represent ${ }^{13} \mathrm{C}$-labeled metabolites of the pentose phosphate pathway (PPP), which were significantly reduced in Flrt2 ${ }^{i \Delta E C}$ mice. The horizontal dotted line represents a $P$ value of 0.05 . Mice were pretreated with ${ }^{13} C_{6}$-glucose to reveal differences in glucose metabolism pathways within the tumor. (B-D) Relative intracellular levels of components of PPP, the glycolytic system, and the TCA cycle, as measured

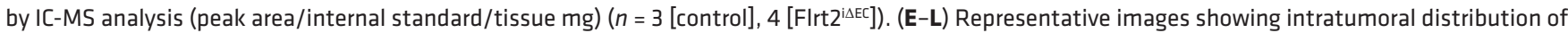
glucose metabolites, as assessed by imaging mass spectrometry (IMS). Representative images for 3 independent experiments are shown. (M) Amount of 2-deoxy-D-glucose (2-DG) leaking from blood vessels and accumulating in tumor cells, as quantified by IC-MS analysis ( $n=3$ [control with 2-DG], 3 [control without 2-DG], 3 [FIrt2 ${ }^{\mathrm{i} \triangle \mathrm{EC}}$ ]). 2-DG is converted to 2DG-6P intracellularly and then accumulates in the tumor (see Supplemental Figure 7). (N-Q) IMS showing the amount and localization of 2DG-6P and 2,3-DPG. Arrowheads indicate colocalization of 2DC-6P and 2,3-DPG in the hemorrhagic area. Representative images for 3 independent experiments are shown. Scale bars: $\mathbf{2} \mathbf{~ m m}$ (E-L and $\mathbf{N}-\mathbf{Q})$. Data are presented as the mean \pm SD. Comparisons between mean values of 2 groups were evaluated using a 2-tailed Student's $t$ test. IMP, inosine monophosphate.

vessels in tumors, thereby protecting them from ROS-mediated damage. Deleting Flrt2 from endothelial cells selectively pruned those abnormalized vessels, leading to uncoupled provision of oxygen and glucose and resulting in reduced tumor intravasation and metastasis without triggering metabolic reprogramming.
Importantly, the relative increase in the number of mature vessels within tumors increased the efficacy of cytotoxic and immunotherapeutic drugs (Figure 9).

Our data suggest there are 2 "complementary" mechanisms of interendothelial cell adhesion. In healthy (quiescent) vessels, 
A
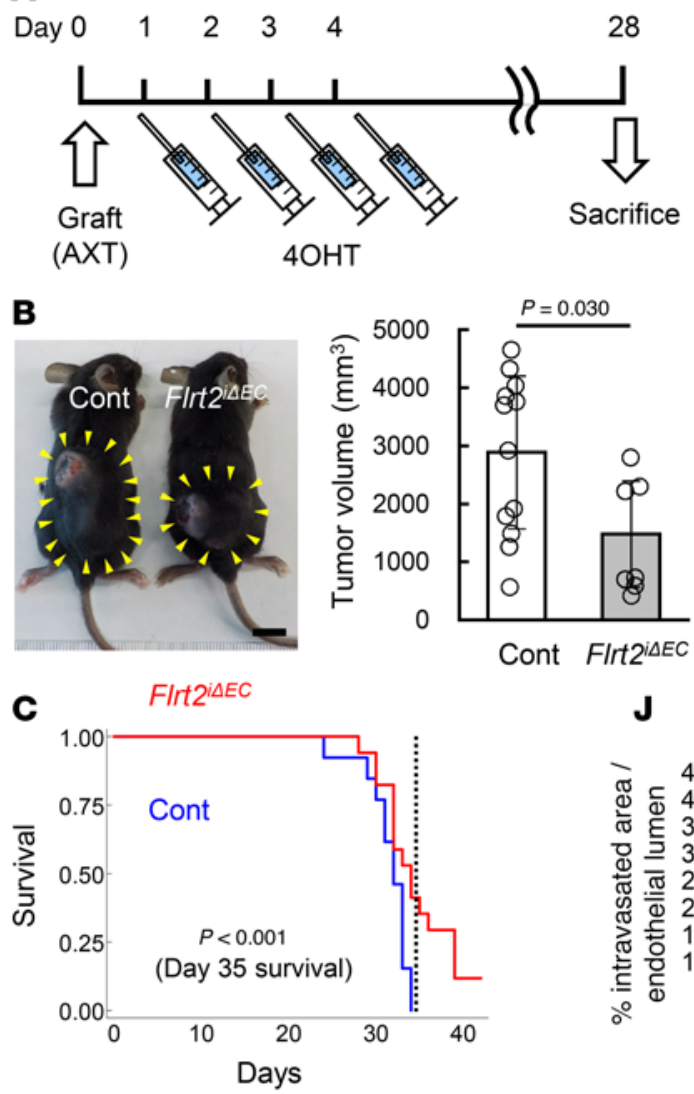

M
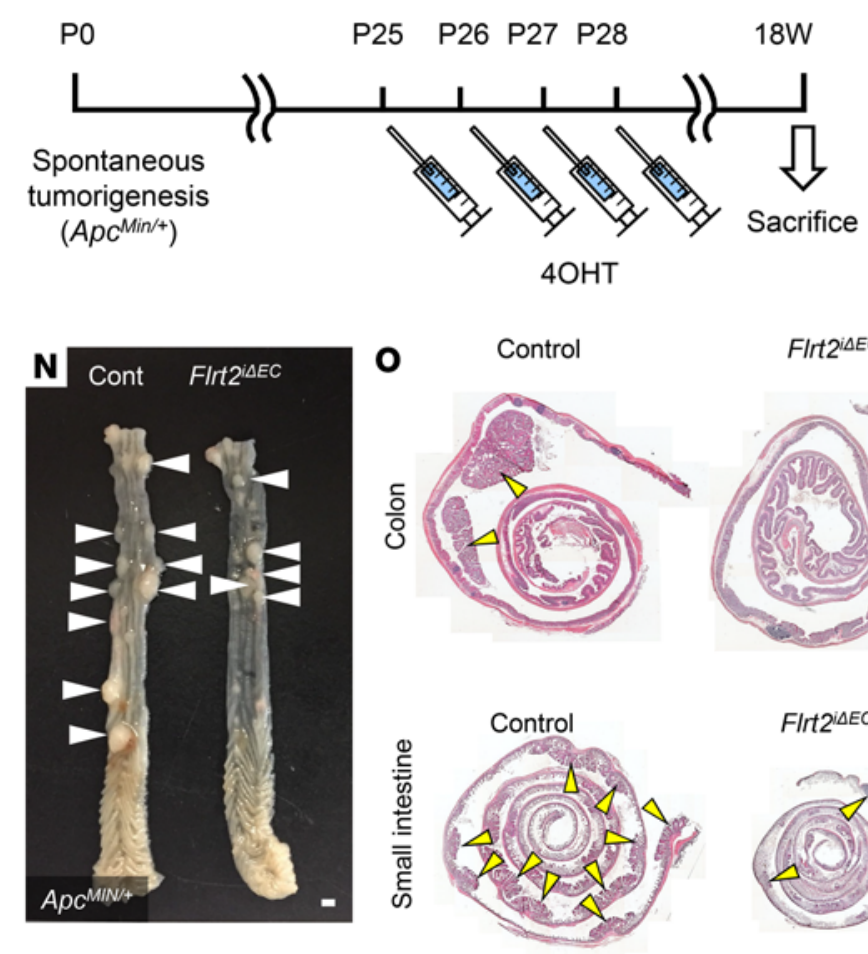
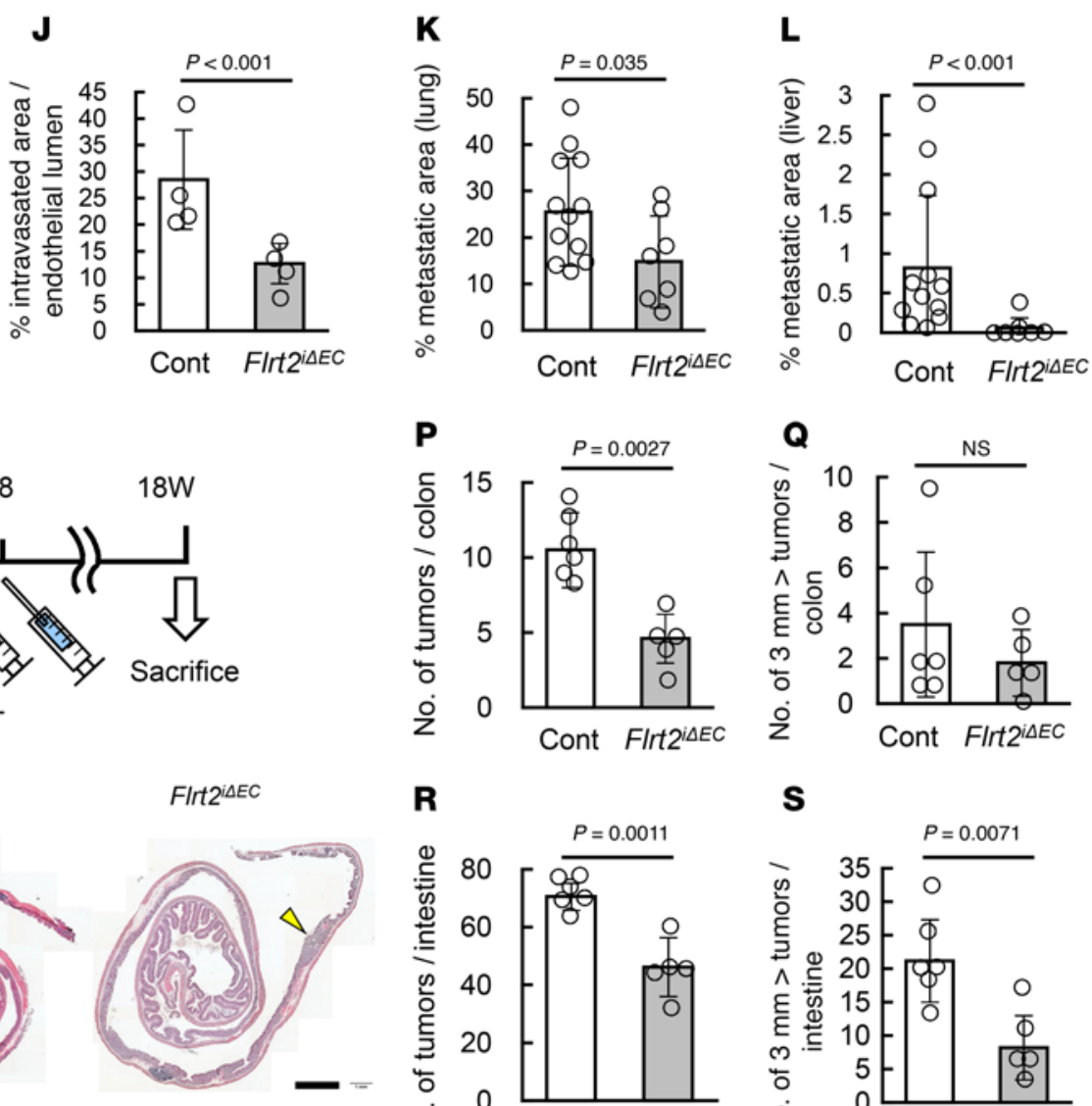

$\mathbf{R}$

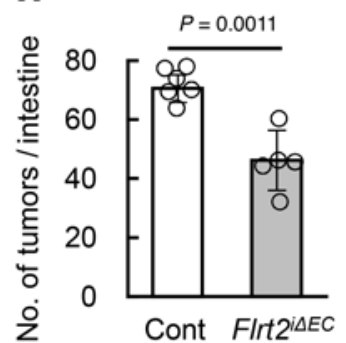

$\mathbf{S}$
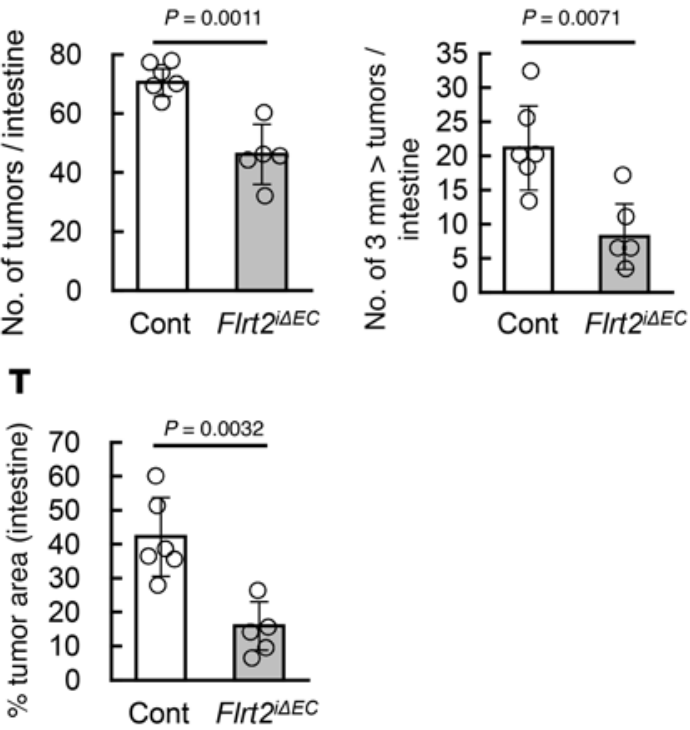
Figure 5. Deletion of Flrt2 prevents tumor metastases and spontaneous tumor formation. (A) Protocol for $40 \mathrm{HT}$ injections and tumor inoculation. (B) Measurement of tumor volume in mice 28 days after transplantation of AXT cells into the back skin (arrowheads) ( $n=12$ [control], 7 [FIrt2 ${ }^{\mathrm{iAEC}}$ ). (C) Kaplan-Meier survival analysis of mice inoculated subcutaneously with AXT cells ( $n=13$ [control], 17 [Flrt2 $\left.{ }^{\mathrm{i \Delta EC}}\right]$ ).

(D-L) Immunohistochemical analysis of primary tumors, lungs, or livers from mice 28 days after transplantation of AXT cells into the back skin ( $n=4$ [control primary], 4 [control lung], 12 [control liver], 7 [FIrt2 ${ }^{\text {isEC }}$

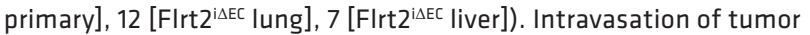
cells (open arrowheads) in primary tumors and their metastasis to distant organs (closed arrowheads) are less pronounced in Flrt2 ${ }^{i \Delta E C}$ mice. (M) Protocol for tamoxifen injection into $A P C^{\mathrm{Min} /+}$ model mice. (N-T) Representative images showing the macroscopic appearance of the colon, along with the sectioned colon and small intestine specimens stained with $\mathrm{H} \& \mathrm{E}\left(n=6\right.$ [control], 5 [Flrt2 ${ }^{i \Delta E C}$ ). $A P C^{\mathrm{Min} / \mathrm{F}} \mathrm{Flrt} 2^{\mathrm{i \Delta EC}}$ mice have fewer and smaller tumors (arrowheads) in both the colon and small intestine than $A P C^{M i n /+}$ mice. Scale bars: $1 \mathrm{~cm}(\mathbf{B}) ; 2 \mathrm{~mm}(\mathbf{F}-\mathbf{I}, \mathbf{N}$, and $\mathbf{0}) ; 50 \mu \mathrm{m}$ (D and $\mathbf{E})$. Data are presented as the mean \pm SD. Comparisons between mean values of 2 groups were evaluated using a 2-tailed Student's $t$ test. Kaplan-Meier curves and the log-rank test were used to compare survival among the groups.

the canonical mechanism, regulated primarily by VE-cadherin, maintains interendothelial cell adhesion and vessel stability even in the absence of FLRT2. Downregulation of VE-cadherin is a well-known hallmark of tumor vessels, which is related to vessel abnormalization $(18,19)$. FLRT2, acting via a noncanonical interendothelial cell adhesion mechanism, compensates for junctional fragility caused by loss of VE-cadherin in abnormalized vessels. The phenotypes of Flrt $2^{i \mathrm{iSC} C}$ mice showed that abnormalized vessels are unable to maintain their architecture and thus collapse if both VE-cadherin and FLRT2 are absent. Oxidative stress is well known to increase internalization of VE-cadherin, resulting in an increase in vessel permeability (39). This was confirmed in our experiments using HUVECs (Figure 1, P and Q). When VE-cadherin is lost due to oxidative stress, increases in the level of FLRT2 may support the interendothelial adhesion under such a condition.

One of the most important aspects of our findings is that FLRT2 is expressed abundantly in the abnormalized vessels of advanced human colon cancers. Expression of many angiogenic molecules is regulated by oxygen, nutrients, or VEGF (1). Here, we show that expression of FLRT2 is dependent on oxidative stress but not on VEGF, a finding that agrees with that of a previous paper (22). In silico screening of human breast cancer cells showed that FLRT2 is one of the most hypermethylated and downregulated genes (40), suggesting epigenetic regulation of FLRT2 in endothelial cells. The regulatory mechanism governing expression of FLRT2, specifically the link between oxidative stress and epigenetic factors, should be comprehensively elucidated.

The opposing actions of FLRT2, repulsive (heterophilic) or adhesive (homophilic), are likely to be context dependent. Our previous in vitro experiments revealed the repulsive effects of FLRT2 when FLRT2 proteins were coated in a stripe manner (16). However, we noticed that some cells stayed on the coated stripes, whereas others migrated toward noncoated areas. These data suggest coexistence of repulsive and adhesive responses for the behavior of HUVECs on FLRT2 proteins. Our current results based on si-RNA-mediated knockdown of the FLRT2 gene, which was not examined in the previous study, might better reflect the adhesive rather than repulsive function of FLRT2. The difference in the function of FLRT2 during development and tumor angiogenesis may be ascribed to differential expression of its receptors. Previous studies show that the function of Unc5b and its other ligand, Netrin1, is highly variable depending on the dose and context (41-43). Netrin-1 acts through Unc5b to function as an endothelial survival factor, whereas unbound Unc5b increases endothelial cell apoptosis (44).

FLRT2 has only a short intracellular region but possesses an extracellular leucine-rich repeat motif that plays a central role in cellular behaviors, including protein-protein interactions, cellular adhesion or repulsion, migration, and growth $(15,45)$. In this respect, an important question remains: do molecular mechanisms related to the homophilic protein interactions of FLRT2 affect endothelial adhesion? Certain cytoskeletal proteins, or other cell adhesion molecules such as integrins, may be affected by homophilic FLRT2 binding, as indicated by a previous study (46). FLRT2 also interacts with growth factor receptors such as FGFR2 (47).

In summary, our findings show that FLRT2 provides noncanonical interendothelial adhesion through homophilic binding, which is exploited by abnormalized vessels within tumors. Our data also suggest that FLRT2 is possibly a novel theranostic molecule with potential utility as an effective and less harmful therapeutic target and/or as a biomarker for the long-term prognosis of patients with colorectal cancer.

\section{Methods}

Mice. Cdh-BAC-Cre ${ }^{E R T 2}$ mice (48), which have been described previously, were mated with Flrt2-flox mice (16), Vegfr2-flox mice (49), Unc5bflox mice (43), and CAG-LSL-GFP mice (50). Sex-matched Cdh5-BAC$\mathrm{Cre}^{E R T 2} \mathrm{Flrt} 2^{f l /+}, \quad$ Cdh5-BAC-Cre ${ }^{E R T 2} V e g f r 2^{f l /+}$, and Cdh5-BAC-Cre $e^{E R T 2}$ $U n c 5 b^{A /+}$ mice were used as littermate controls. $A P C^{\mathrm{Min} /+}$ mice were obtained from The Jackson Laboratory (stock no. 002020). Mutant mice were crossed $>10$ times with C57BL/6J mice. Both sexes were used for the analysis of the phenotypes of mutant mice.

Tumor models. B16 mouse melanoma cells were cultured in DMEM containing $10 \%$ FBS. Cells $\left(2 \times 10^{6}\right)$ were implanted subcutaneously into the backs of 4 - to 6-week-old male mice. AXT mouse osteosarcoma cells were cultured in Iscove's modified Dulbecco's medium (Invitrogen) supplemented with 10\% FBS and were implanted similarly. Tumors were measured daily with a caliper volume calculated using the following formula: $V=$ tumor length $\times$ width $\times$ height $/ 2$. For Cre induction in $C d h-B A C-C r e^{E R T 2}$ mice, 100 $\mu \mathrm{l} 4 \mathrm{OHT}$ dissolved in oil at $5 \mathrm{mg} / \mathrm{mL}$ was injected into the inguinal fat according to the schedules shown in the figures. For some experiments, mice were injected intraperitoneally with $2.5 \mathrm{mg} / \mathrm{kg}$ per g bodyweight cisplatin dissolved in $100 \mu \mathrm{DMSO}$ or with the same volume of DMSO as a control, after tumors reached $30 \mathrm{~mm}^{3}$. For PD1 blockade, mice were injected intraperitoneally with $10 \mathrm{mg} / \mathrm{kg}$ per g bodyweight of an anti-PD1 mAb (BioXCell; BE0146) dissolved in $100 \mu \mathrm{l}$ PBS; infections were given every 3 days, starting 6 days after tumor cell inoculation.

Wound healing assay. On 4 consecutive days, male mice (aged 4-6 weeks) were injected with $100 \mu \mathrm{l} 4 \mathrm{OHT}$ dissolved in oil at $5 \mathrm{mg} /$ $\mathrm{mL}$ (injections were administered into the inguinal fat). At 10 days 


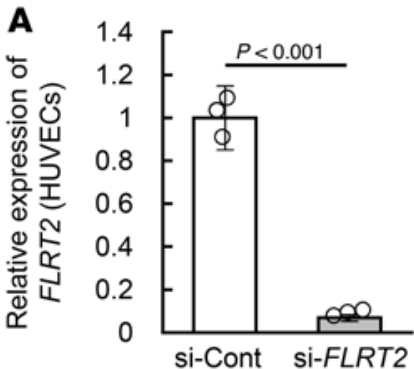

C

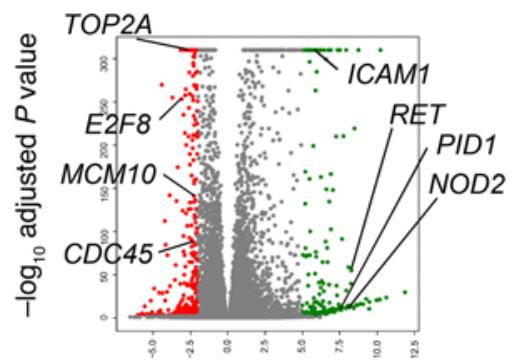

Significant down in $\log _{2} \mathrm{FC} \quad$ Significant up in
si-FLRT2
si-FLRT2

\section{E}

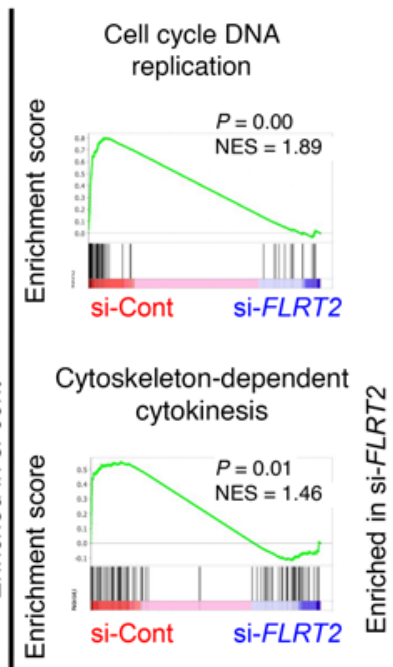

\section{Protein localization to} cytoskeleton

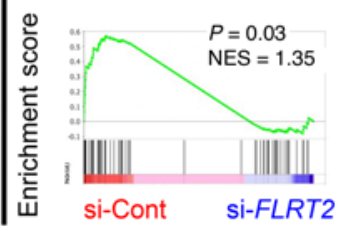

B

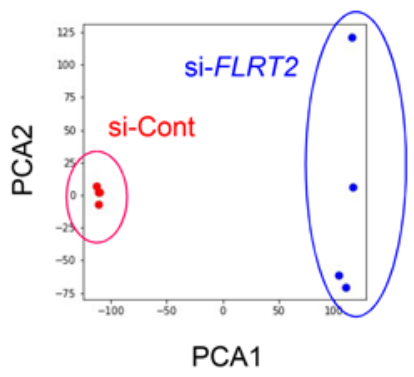

D

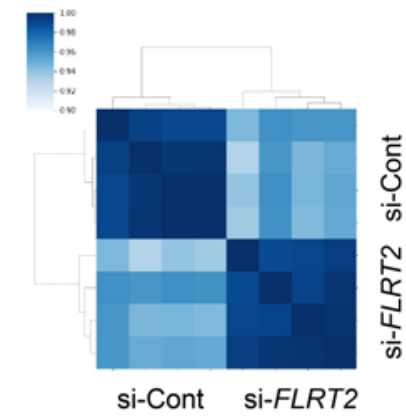

F Cell cycle DNA replication

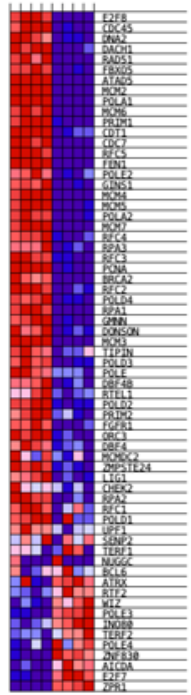

Positive regulation of stress activated protein kinase

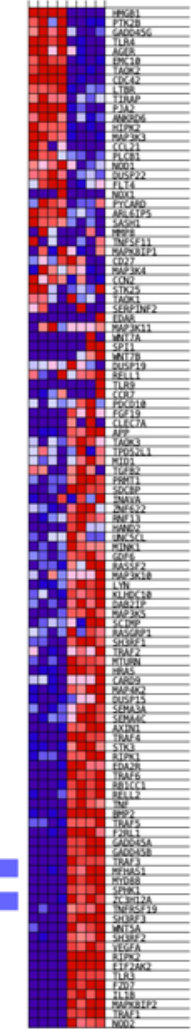

G IPA (disease and function)

Positive regulation of stress activated protein kinase

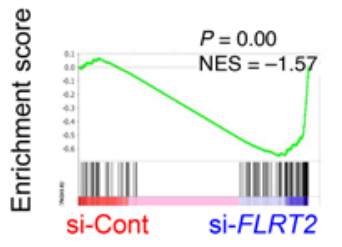

Organismal injury and abnormalities

Cellular assembly and organization DNA replication, recombination, and repair Tissue morphology Infectious diseases Gastrointestinal disease Connective tissue disorders Immunological disease Inflammatory disease Skeletal and muscular disorders Reproductive system disease Cell death and survival Negative regulation of cell cell adhesion

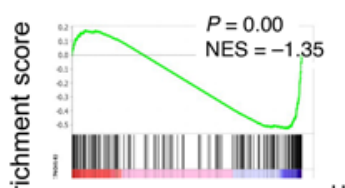

Dermatological diseases and conditions Organismal survival Inflammatory response Cellular movement Endocrine system disorders si-Cont si-FLRT2 Hematological system development and function Immune cell trafficking Hematological disease Antimicrobial response Respiratory disease Neurological disease Hepatic system disease Tissue development Metabolic disease Organismal development Cell-to-cell signaling and interaction reactive oxygen species metabolic process

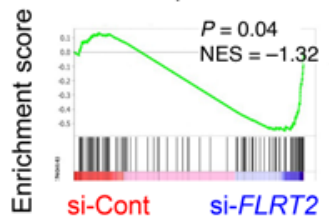

Figure 6. FLRT2 silencing affects genes related to oxidative stress and intercellular adhesion. (A) RT-PCR analysis of HUVECs treated with siRNA ( $n=3$ each). (B) Principal component analysis of RNA-Seq data from HUVECs treated with si-Cont or si-FLRT2. (C) Volcano plot of genes differentially expressed by HUVECs treated with si-Cont or si-FLRT2. Genes significantly upregulated or downregulated $(q<0.01, \operatorname{logFC}>5$ or $<-2)$ in si-FLRT2-treated HUVECs are marked in red or green, respectively. (D) Correlation matrix showing the Pearson correlation coefficients for each RNA-Seq experiment. (E and F) GSEA plots and heat maps of up- or downregulated genes in si-FLRT2-treated HUVECs. (C) Ingenuity Pathway Analysis (IPA) of differentially expressed genes between HUVECs treated with si-Cont or si-FLRT2. Terms underscored by red lines are related to the phenotype in Flrt2 ${ }^{i \Delta E C}$ mice. Data are presented as the mean \pm SD. Comparisons between mean values of 2 groups were evaluated using a 2-tailed Student's $t$ test. 

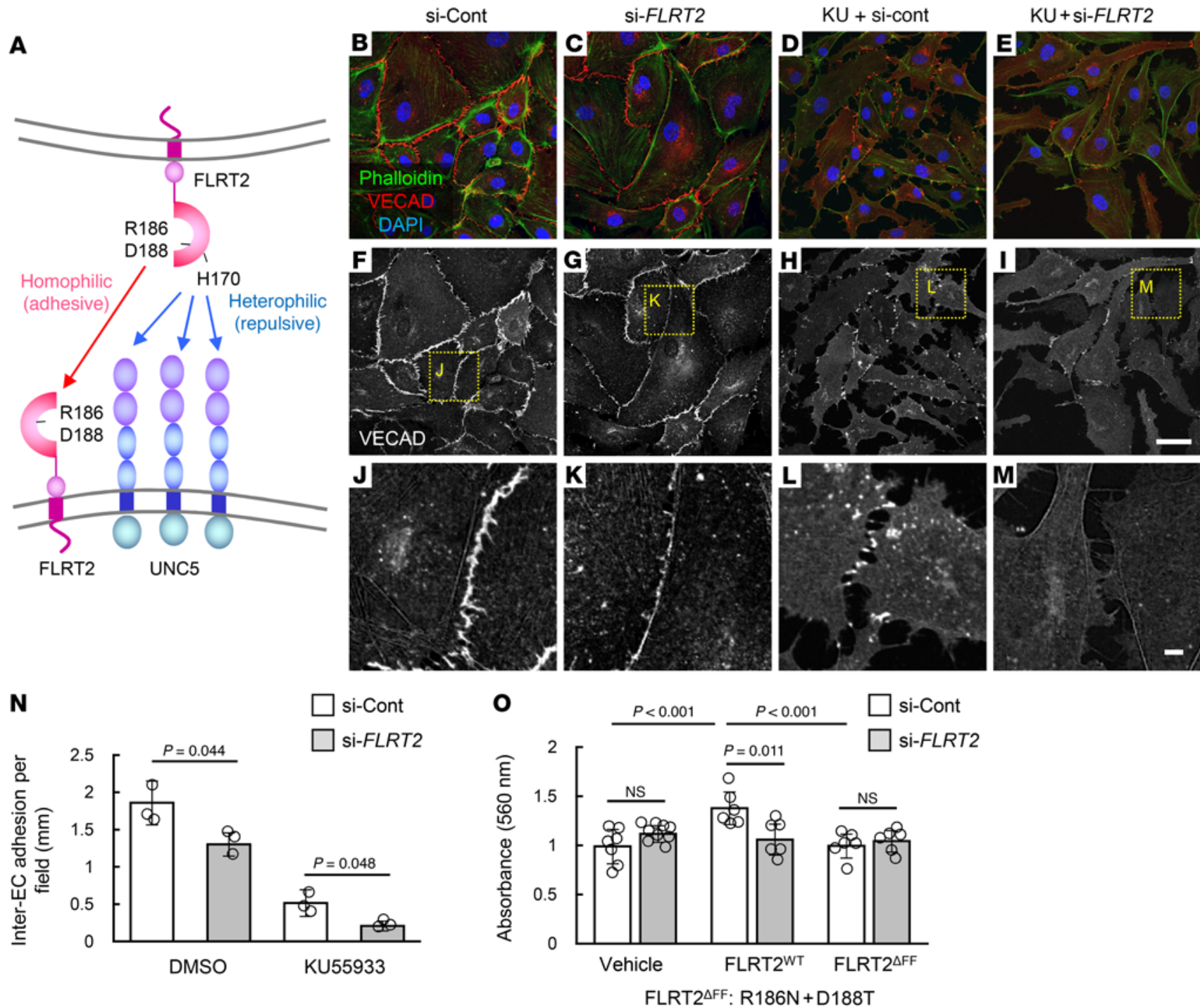

Figure 7. Intercellular adhesion via homophilic binding of FLRT2 protects ECs against oxidative stress. (A) A scheme depicting the wide-ranging actions of FLRT2. (B-N) Immunocytochemical analysis of HUVECs, and analysis of VECAD+ interendothelial cell (EC) adhesion ( $n=3$ each). (0) Adhesion of EC harboring recombinant FLRT2 proteins ( $n=7$ [Vehicle si-Cont], 8 [Vehicle si-FLRT2], 6 [FLRT2WT si-Cont], 6 [FLRT2WT si-FLRT2], 6 [FLRT2 $\Delta$ FF si-Cont], 6 [FLRT2 $\Delta$ FF si-FLRT2]). Data are presented as the mean \pm SD. Scale bar: $50 \mu \mathrm{m}$ (B-I); $5 \mu \mathrm{m}$ (J-M). Comparisons between mean values of 2 groups were evaluated using a 2-tailed Student's $t$ test. Comparisons among multiple groups (0) were evaluated using 2-way ANOVA followed by Bonferroni's multiple-comparison test.

after $4 \mathrm{OHT}$ injection, a full-thickness wound on the dorsal area was made in the shape of a circle measuring $10 \mathrm{~mm}$ in diameter. At 7 days after surgery, the wounds were resected (including a margin of normal surrounding skin) and fixed in 4\% PFA. Paraffin sections were prepared as described previously and processed for Masson's trichrome staining or fluorescent IHC.

Preparation of whole-mount retinal samples. Enucleated eyes were fixed for 20 minutes in 4\% PFA in PBS and then dissected as described previously (48). Retinal cups were post fixed for 20 minutes and were stained as described below.

Ischemic retinopathy model. $\mathrm{P} 8$ mice with nursing mothers were maintained for 3 days in an atmosphere containing $85 \%$ oxygen and then placed back in room air as previously described (21). In some experiments, intraperitoneal administration of $\mathrm{N}$-acetyl-Lcysteine (MilliporeSigma) dissolved in PBS (injection volume $0.5 \mathrm{~g}$ $\mathrm{kg} /$ body weight/d) was performed daily, from P11 to P15, in the ischemic retinopathy model.

Generation of Flrt $2^{\text {R186N+D188T }}$ mice. Using the Optimized CRISPR design tool (Massachusetts Institute of Technology; http://crispr.mit. edu/), a CRISPR guide RNA (AGACTTGCAAGAGCTGAGAG) and an ssOligoDNA were designed to replace guanine 557 and alanine 558 in mouse Flrt2 withalanine and cytosine respectively (c.557_558GA $>\mathrm{AC}$ ), resulting in an amino acid substitution (p186Arg $>$ Asn). In addition, guanine 562 and alanine 563 were replaced with alanine and cytosine (c.562_563GA $>$ AC) to yield a second amino acid substitution (p188Asp $>$ Thr). Next, gRNA-Cas9-expressing vectors (pX330Flrt2-gRNA) were constructed. These gRNA-Cas9 vectors (30 $\mathrm{ng} / \mu \mathrm{l})$, along with ssOligoDNA $(10 \mathrm{ng} / \mu \mathrm{l})$, were comicroinjected into the pronuclei of fertilized mouse eggs. Surviving eggs were implanted into the oviducts of pseudopregnant foster mothers. Mice 
A
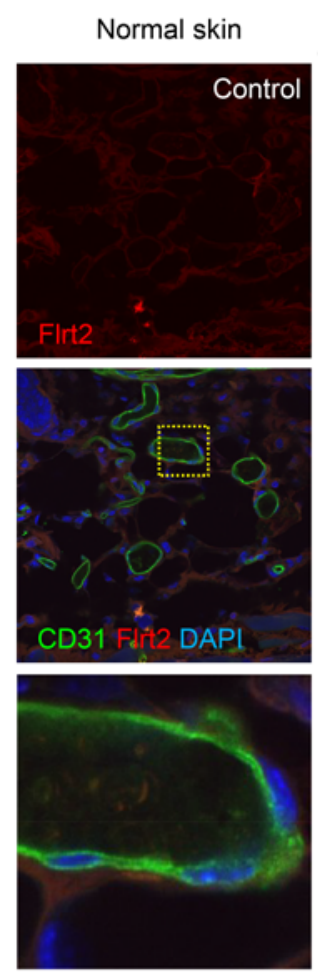

AXT model (day 28)

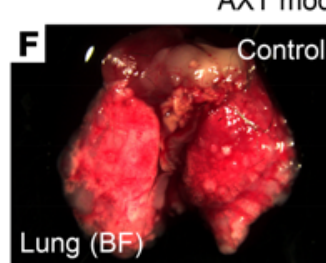

H
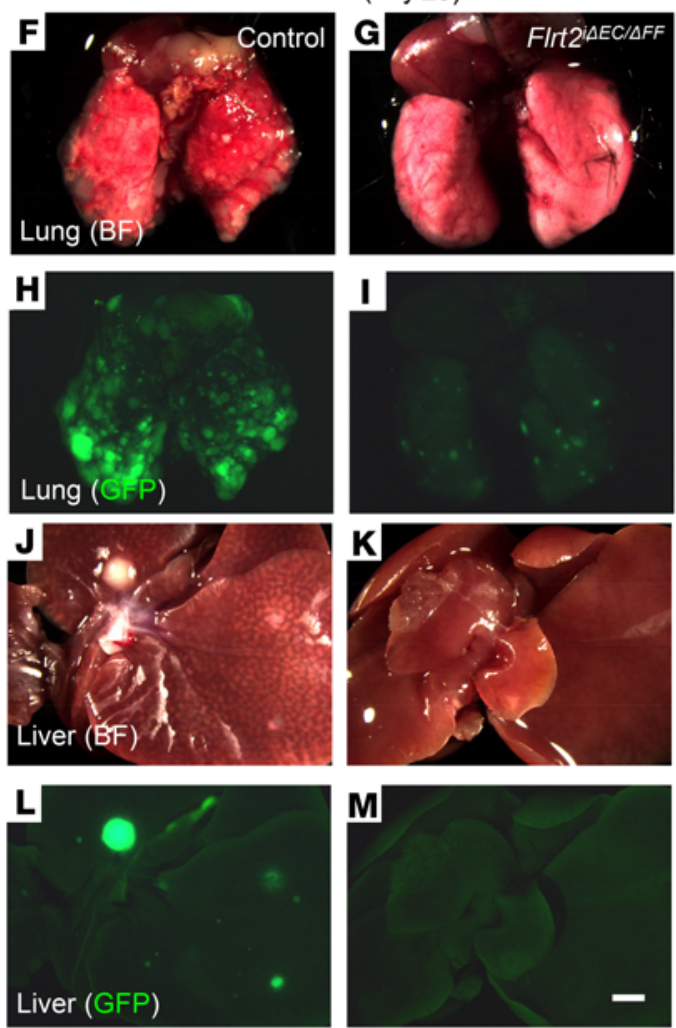

M

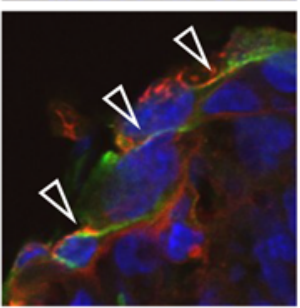

B

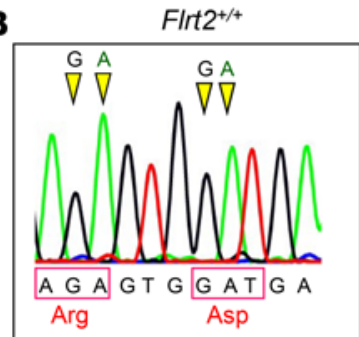

C
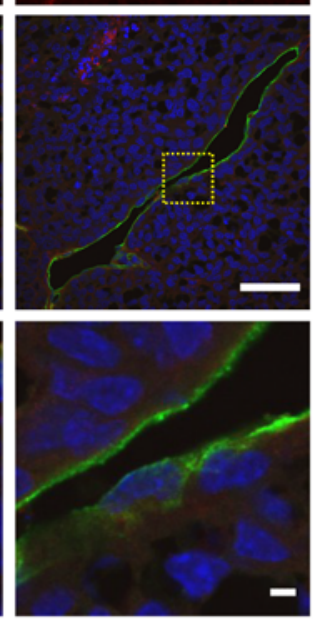

$\mathbf{N}$

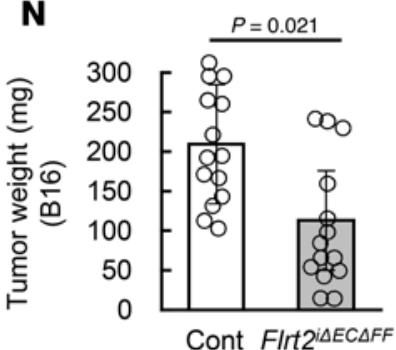

$\mathbf{P}$

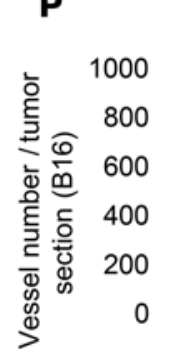

$P=0.035$

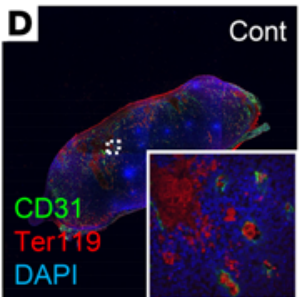

o

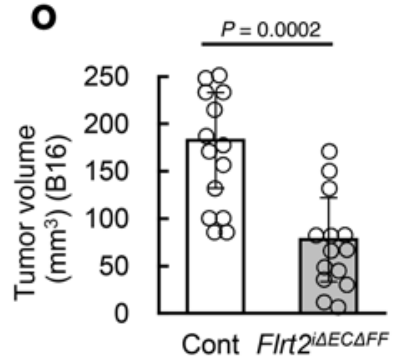

$\mathbf{Q}$

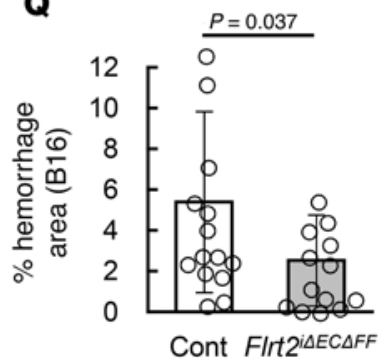

$\mathbf{R}$

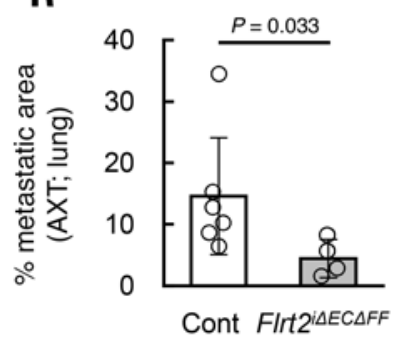

FIrt2 R186N+D188T/R186N+D188T

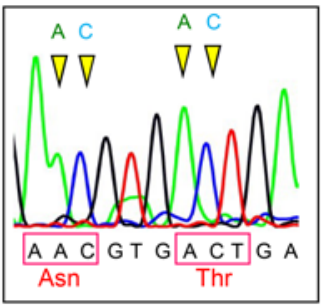

B16

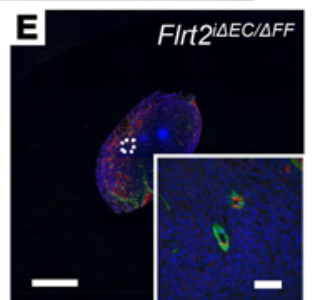

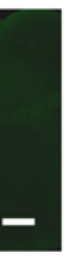


Figure 8. Homophilic binding of FLRT2 constitutes tumor-specific interendothelial adhesion. (A) Immunohistochemical analysis of sectioned tumors from mice 10 days after transplantation of B16 cells into the back skin. Arrowheads indicate interendothelial localization of FLRT2 proteins. Representative images for 3 independent experiments are shown. (B) Nucleotide sequences showing mutations in the Flrt2 ${ }^{R 186 N+D 188 T}$ allele. Representative chromatograms for 3 independent experiments are shown. (C-E) Macroscopic view and immunohistochemical analysis of sectioned tumors from mice 10 days after transplantation of B16 cells into the back skin. (F-M) Macroscopic appearance of lungs and livers from mice 28 days after transplantation of AXT cells into the back skin. (N-Q) Quantification for mice 10 days after transplantation of AXT cells into the back skin ( $n=14$ each). ( $\mathbf{R}$ and $\mathbf{S}$ ) Quantification for mice 28 days after transplantation of AXT cells into the back skin ( $n=6$ [control R], 4 [Flrt2i $\Delta E C$ R], 5 [control S], 4 [FIrt2i $\Delta \mathrm{EC} \mathrm{S}$ ]). Data are presented as the mean \pm SD. Scale bar: $2 \mathrm{~mm}$ (C-M); $50 \mu \mathrm{m}$ (top 2 rows in $\mathbf{A}$, insets of $\mathbf{D}$, and $\mathbf{E}$ ); $5 \mu \mathrm{m}$ (bottom row in A). Comparisons between mean values of 2 groups were evaluated using a 2-tailed Student's $t$ test.

born to the foster mothers were genotyped by direct sequencing using the following primer: Flrt2FF-SEQ, 5'-TGGCAACCAGTTGGATGAGTTC-3'. PCR products were amplified using 2 primers: Flrt2FF-F, 5'-GCACACTGTCTACCTTTATGGCAAC-3', and FLRT2FF-R, 5' -TGGTTATCCTGCAAGTAGAGCCTG-3'.

Preparation of tissue sections and whole-mount samples. Surgically dissected tissues were fixed overnight in $4 \%$ paraformaldehyde (PFA) in PBS. Frozen- or paraffin-embedded sections from those samples were used for immunohistochemical analysis. Before sectioning primary tumors derived from AXT cells, tumors were decalcified by overnight immersion in 0.5 M EDTA. All tumor samples were sectioned ( $14 \mu \mathrm{m}$ thick for frozen samples or $3 \mu \mathrm{m}$ for paraffinembedded samples) at the plane of the maximum cut surface. To prepare whole-mount samples, tissues were dissected and fixed overnight in $4 \%$ PFA in PBS. All sections and whole-mount tissues were stained as described below.

Immunostaining. IHC of whole-mount samples or tissue sections was performed as described previously (51). The following primary monoclonal antibodies were used: hamster anti-CD31 (Chemicon, Temecula; MAB1398Z; 1:1,000), $\alpha$-SMA-Cy3-conjugated (MilliporeSigma; C6198; 1:500), FLRT2 (R\&D Systems; MAB2877; 1:500), VE-cadherin (Santa Cruz, sc-9989; 1:200), Ter119 (R\&D Systems, MAB1125; 1:500), and Ki67 (Novus, NB600-1252; 1:500). The following primary polyclonal antibodies were used: anti-GFP-Alexa Fluor 488-conjugated (Molecular Probes,; A21311; 1:500), Collagen IV (Cosmo Bio, LSL-LB-1407; 1:500), and FLRT2 (R\&D Systems, AF2877; 1:1,000). The secondary antibodies were Alexa Fluor 488conjugated IgG (Molecular Probes, A11034, A11006, A11055; 1:500) and Cy3/Cy5 DyLight549/DyeLight649-conjugated IgG (Jackson ImmunoResearch; 711-165-152, 112-165-167, 127-165-160, 711-605152, 112-605-167, 127-605-160, respectively; 1:500). Alexa Fluor 647-conjugated phalloidin (Invitrogen, A22287; 1:40) was used to visualize F-actin. To detect hypoxic cells, the Hypoxyprobe-1 Plus Kit (Chemicon, HP2-100) was used. Briefly, $60 \mathrm{mg} / \mathrm{kg}$ pimonidazole was injected intraperitoneally into mice 30 minutes before euthanasia. Sections were stained with Hypoxyprobe Mab1-FITC. For nuclear staining, specimens were treated with DAPI (Molecular Probes, D-1306). For angiography, $50 \mu \mathrm{l}$ FITC-dextran $(10 \mathrm{mg} / \mathrm{mL}$; MW
$2000 \mathrm{kDa}$; MilliporeSigma) in PBS was injected into the left cardiac ventricle and allowed to circulate for 3 minutes.

Confocal microscopy. Fluorescence images were obtained under a confocal laser scanning microscope (Olympus, FV1000). Quantification of cells or parameters of interest was conducted using 3 images, each with a $1270 \times 1270 \mu \mathrm{m}$ field of view, per sample (to count Ki67 ${ }^{+}$ cells and vessels with tumor cell intravasation and to identify $\mathrm{SMA}^{+}$ vessels), or entire sections of tumor, lung, and liver (to count tumor vessels and to measure hemorrhagic, perfused, hypoxic, metastatic, and vascularized areas). Image J software (NIH) was used for quantification of the indicated areas.

Sample preparation for ${ }^{13} \mathrm{C}_{6}$-glucose metabolic pathway tracing analysis. To trace the metabolic pathways of cancer cells, ${ }^{13} \mathrm{C}_{6}$-glucose (1 mg/body weight [g]) was injected intraperitoneally into mice 30 minutes before resecting tumors (on the tenth day after implantation). Dissected tumors were frozen immediately and stored at $-80^{\circ} \mathrm{C}$ until use. Tumor tissues were used for both metabolome analysis and IMS. Metabolite extraction for metabolome analysis was performed as described previously (52). Briefly, frozen tissue blocks and internal control compounds (2-morpholinoethanesulfonic acid) were homogenized in ice-cold methanol (500 $\mu \mathrm{l})$ using a manual homogenizer (Finger Masher; AM79330), followed by addition of an equal volume of chloroform and 0.4 times the volume of ultrapure water (LC/MS grade, Wako). The suspension was then centrifuged at $15,000 \mathrm{~g}$ for 15 minutes at $4^{\circ} \mathrm{C}$. After centrifugation, the aqueous phase was filtered using an ultrafiltration tube (Human Metabolome Technologies; Ultrafree-MC, UFC3 LCC NB). The filtrate was concentrated in a vacuum concentrator (Thermo Fisher Scientific; SpeedVac), dissolved in $50 \mu$ ultrapure water, and used for metabolome analysis.

Metabolome analysis. For metabolome analysis focused on glucose metabolic central pathways, namely glycolysis, the TCA cycle, and the PPP, anion metabolites were measured using an Orbitraptype mass spectrometer (Thermo Fisher Scientific; Q-Exactive Focus) connected to a high-performance ion chromatography (IC) system (Thermo Fisher Scientific; ICS-5000+), which enabled performance of highly selective and sensitive metabolite quantification owing to the IC separation and Fourier Transfer MS principle (53). The IC device was equipped with an anion electrolytic suppressor (Thermo Fisher Scientific; Dionex AERS 500), which converts the potassium hydroxide gradient into pure water before the sample enters the mass spectrometer. Separation was performed using a Dionex IonPac AS11-HC, $4 \mu \mathrm{m}$ particle size column (Thermo Fisher Scientific). The IC flow rate was $0.25 \mathrm{~mL} / \mathrm{min}$, supplemented post-column with a $0.18 \mathrm{~mL} / \mathrm{min}$ makeup flow of methanol. The potassium hydroxide gradient conditions for IC separation were as follows: 1-100 $\mathrm{mM}$ (0-40 $\mathrm{min}), 100 \mathrm{mM} \mathrm{(40-50} \mathrm{min),} \mathrm{and} 1 \mathrm{mM}$ (50.1-60 $\mathrm{min})$, at a column temperature of $30^{\circ} \mathrm{C}$.

Matrix coating and MALDI-IMS acquisition. MALDI imaging analyses were performed as described previously (54). Briefly, thin sections $(8 \mu \mathrm{m})$ of tumor were prepared with a cryomicrotome (Leica Microsystems; CM3050). Sections were attached onto indium tin oxide-coated glass slides (Bruker Daltonics $\mathrm{GmbH}$ ) and coated with 9-aminoacridine as the matrix $(10 \mathrm{mg} / \mathrm{mL}$, dissolved in $80 \%$ ethanol) by manual spraying with an artistic brush (Procon Boy FWA Platinum, Mr. Hobby). The matrix was applied simultaneously to multiple sections to maintain consistent analyte extraction and 


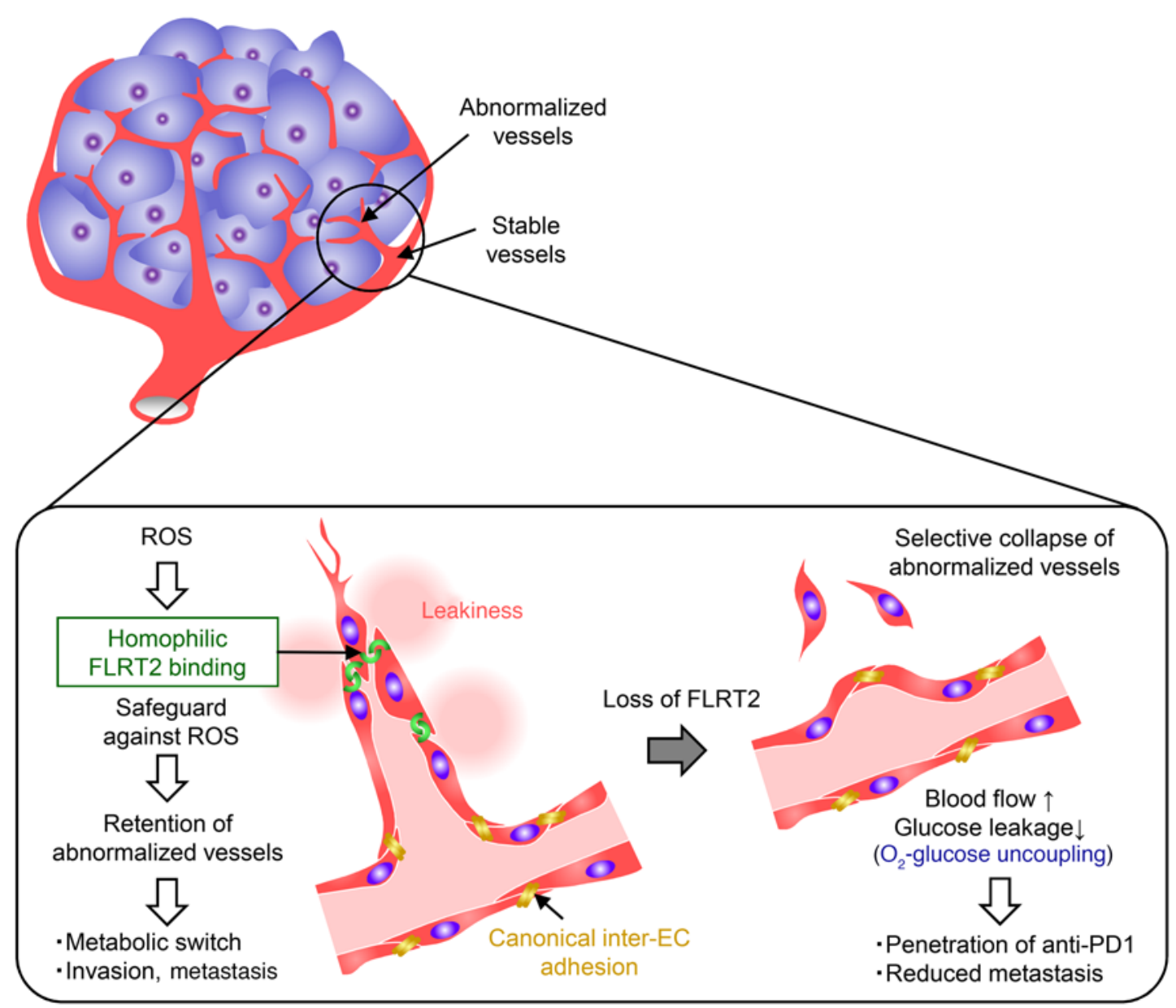

Figure 9. Proposed model for the role of FLRT2 in tumor angiogenesis. Proposed model for the role of FLRT2 as a safeguard against ROS in abnormalized tumor vessels, and the consequence of FLRT2 deletion.

cocrystallization conditions. MALDI imaging was performed using an Ultraflextreme MALDI-TOF/TOF mass spectrometer equipped with an Nd:YAG laser. Data were acquired in the negative reflection mode, with raster scanning at a pitch distance of $50 \mu \mathrm{m}$. Each spectrum was the result of 300 laser shots per data point. For TOF/ TOF measurement, signals between $\mathrm{m} / z 50$ and 1000 were collected. Image reconstruction for both procedures was performed using FlexImaging 4.1 software (Bruker Daltonics). Molecular identification was based on an accurate $m / z$ value derived from FT-ICR-MS data and previous studies (54).

${ }^{13} \mathrm{C}_{6}$-glucose metabolic pathway-tracing analysis. 2-DG (1 mg/body weight $[\mathrm{g}]$ ) was injected intraperitoneally 30 minutes before resecting tumors (on the tenth day after implantation). The amount of 2DG-6P was quantified by IC-MS, and the tissue distribution was assessed by MALDI-IMS as described above.

RT-PCR analysis. Total RNA was prepared from cultured cells, or from $\mathrm{CD} 1^{+}$endothelial cells isolated from the B16 tumors using collagenase and dispase, and purified using Dynabeads (Veritas). Reverse transcription was performed using Superscript III (Invitrogen). cDNA samples were subjected to PCR amplification using the following primers: (a) mouse Flrt2, 5'-AGACAAGGCTGCCAGATTACA-3' and 5'-TAAAATGCAACGTGATGGGG-3'; (b) mouse $\beta$ actin, $5^{\prime}$-ATGGATGACGATATCGCTGC-3' and $5^{\prime}$-AGCACTGT-
GTTGGCATAGAG-3'. Quantitative PCR assays were performed on an ABI 7500 Fast Real-Time PCR System using TaqMan Fast Universal PCR master mix (Applied Biosystems) and TaqMan Gene Expression Assay Mix with human FLRT2 (Hs00544171_s1) and FLRT3 (Hs01922255_s1). A human $\beta$-ACTIN (Hs00357333_gl) assay mix served as an endogenous control. Data were analyzed using 7500 Fast System SDS Software 1.3.1.

Cell culture. HUVECs were cultured in EGM-2 medium (Takara-Bio) at $37^{\circ} \mathrm{C} / 20 \% \mathrm{O}_{2}$. In some experiments, $10 \mu \mathrm{M} \mathrm{KU} 55933$ (Calbiochem) dissolved in DMSO or $400 \mu \mathrm{M} \mathrm{H}_{2} \mathrm{O}_{2}$ was added to the culture medium, and cells were cultured for 24 hours.

RNA interference. For the RNA interference experiments, HUVECs were washed once with OptiMEM (Thermo Fisher Scientific) and transfected with $40 \mathrm{nmol} / \mathrm{L}$ siRNA Duplex (QIAGEN) using $6 \mu \mathrm{l} / \mathrm{mL}$ Lipofectamine RNAiMAX (Invitrogen) in OptiMEM. After 4 hours, the transfection medium was removed, and complete culture medium was added. Cells were cultured for a further 48 hours before use in in vitro assays. FlexiTube siRNA SIO3061226 for FLRT2 (5'-CACGGUCUACCUGUAUGGCAA-3'; QIAGEN) was used to knockdown FLRT2. A human siRNA-negative control duplex oligonucleotide (5'-CUUACGCUGAGUACUUCGATT-3', 5'-UCGAAGUACUCAGCGUAAGTT-3'; MilliporeSigma) was used as the negative control. 
RNA-Seq analysis. The library for sequencing was prepared according to the manufacturer's protocol (KAPA Biosystems; KAPA RNA HyperPrep Kit). Next, $2 \times 150$ bp pair-end sequencing was carried out using Illumina Hiseq. Sequencing data were converted into the fastq format using bcl2fastq software. The sequence quality was checked by FastQC software, and adapter sequences were eliminated by Trimmomatic. The sequence data were processed using the STAR-RSEM-DESeq2 pipeline. DEGs between HUVECs treated with si-Control and those treated with si-FLRT2 were extracted by the Wald test using DESeq2 packages. TPM was used for the following analyses. The correlation matrix was calculated using Pearson correlation. Principle component analysis was performed using the scikit-learn package. A volcano plot was constructed using the bioinfokit package. GSEA was performed using the ontology gene sets in the Molecular Signatures Database (http://www.gsea-msigdb.org/ gsea/msigdb/). IPA was performed to identify the most significant pathways for the DEGs $(q<0.01, \operatorname{logFC}<-2$ or $>5)$ in si-FLRT2treated HUVECs compared with si-Control-treated cells. In brief, we uploaded the gene list file containing gene symbols and $\log \mathrm{FC}$; we then adjusted $P$ values to the IPA and performed the core analysis. RNA-Seq data generated during this study are available at GEO (accession GSE179836).

Adhesion assay. Adhesion to ECM molecules was examined using a colorimetrically formatted CytoSelect 48-Well Cell Adhesion Assay Kit (Cell Biolabs). Briefly, cultured cells were suspended in serum-free medium at a concentration of $1 \times 10^{5}$ cells $/ \mathrm{mL}$. Next, $150 \mu \mathrm{l}$ of the cell suspension was added to each well and incubated for 60 minutes. Then, nonadherent cells were washed out, and $200 \mu$ l Cell Stain Solution (Cell Biolabs) was added to stain adherent cells. After washing away this solution, $200 \mu$ l Extraction Solution (Cell Biolabs) was added to each well, and the extracted sample was transferred to a 96-well microtiter plate to measure the optical density at $560 \mathrm{~nm}$. Adhesion assays for the FLRT2 protein were performed using recombinant wild-type or mutant FLRT2 proteins. These proteins were diluted to the indicated concentration in PBS and coated onto 96-well flat-bottomed nontissue culture-treated polystyrene plates for 1 hour. Nonspecific sites were blocked for 1 hour at room temperature with $10 \mathrm{mg} / \mathrm{mL}$ BSA. After blocking, wells were washed with PBS. Next, $1 \times 10^{5}$ HUVECs in $0.2 \mathrm{~mL}$ medium were added to each coated well. Plates were incubated at $37^{\circ} \mathrm{C}$ for 2 hours. Nonadherent cells were removed by washing with PBS, and the remaining cells were fixed for 30 minutes at room temperature in $4 \%$ PFA. Fixed cells were stained for 30 minutes with $0.5 \%$ crystal violet in $25 \%$ methanol and then solubilized in $1 \%$ SDS. OD values were measured.

Human studies. A cohort of 47 human malignant patients with colorectal cancer was analyzed to determine the expression pattern of FLRT2 in tumors. The 5-year RFS of another cohort (66 patients) with stage II/III cancer was analyzed alongside histological examinations. Sections ( $4 \mu \mathrm{m}$ thick) of formalin-fixed and paraffinembedded tissues were used for histological analysis. After deparaffinization and rehydration, sections were autoclaved at $121^{\circ} \mathrm{C}$ for 5 minutes in citrate buffer for antigen retrieval before immunostaining. To quantify FLRT2 expression, a $2200 \times 1700$ $\mu \mathrm{m}$ field of view in each section was examined under a light microscope, and the IHC score was determined objectively by 2 blinded researchers using the following formula: IHC score $=1 \times$ (mildly positive vessels $[\%])+2 \times($ moderately positive vessels $[\%])+3 \times$ (highly positive vessels [\%]).

Statistics. All results are expressed as the mean \pm SD. Comparisons between mean values of 2 groups were evaluated using a 2-tailed Student's $t$ test. Comparisons among multiple groups were evaluated using 2-way ANOVA followed by Bonferroni's multiple-comparison test. Kaplan-Meier curves and the log-rank test were used to compare survival among the groups. Pearson's correlation analysis was performed to determine correlations among groups. All calculations were performed using Stata 11.2 (Stata Corporation). $P$ values of less than 0.05 were considered significant.

Study approval. Animal use and care were approved by the Institutional Animal Care and Use Committee of Keio University, and all experiments were performed in accordance with the Guidelines of Keio University for Animal and Recombinant DNA experiments. The human study was approved by the institutional ethics committee of Keio University Hospital (approval no. 20150051). A written informed consent was received from participants prior to their inclusion in the study.

\section{Author contributions}

Y Kitagawa and Y Kubota designed the experiments. TA, Y Kitagawa, KO, YS, and ITN performed the experiments. TA, DK, KO, YS, ITN, Y Kido, and Y Kubota analyzed the data. HS, SN, DYL, ES, and SY provided experimental materials. KS, MS, ES, and SY edited the manuscript. TA and Y Kubota wrote the paper.

\section{Acknowledgments}

We thank Rüdiger Klein of the Max Planck Institute of Neurobiology, Martinsried, Germany, for providing the Flrt2-flox mice. This work was supported by Grants-in-Aid for Specially Promoted Research from the Ministry of Education, Culture, Sports, Science, and Technology of Japan (18H05042, 18K19553, and 19H03397); by AMED-PRIME (JP2Ogm6210017h0002 and 21gm6210017h0003); and by Japan Science and Technology Agency (Moonshot R\&D) (JPMJMS 2024) and by research grants from the following: Inamori Foundation, Kao Foundation for Arts and Culture, Takeda Science Foundation, Mochida Memorial Foundation, Mitsubishi Foundation, Cell Science Research Foundation, SENSHIN Medical Research Foundation, Sumitomo Foundation, Daiichi Sankyo Foundation of Life Science, Naito Foundation, Uehara Memorial Foundation, and Toray Science Foundation.

Address correspondence to: Yoshiaki Kubota, Department of Anatomy, Keio University School of Medicine, 35 Shinanomachi, Shinjuku-ku, Tokyo 160-8582, Japan. Phone: 81.3.5315.4358; Email: ykubo33@a3.keio.jp. Or to: Satoru Yamagishi, Organ and Tissue Anatomy, Hamamatsu University School of Medicine, 1-20-1, Handayama, Higashi-ku, Hamamatsu, Shizuoka, 431-3192, Japan. Phone: 81.53.435.2290; Email: yamagish@hama-med.ac.jp. 
1. Potente M, et al. Basic and therapeutic aspects of angiogenesis. Cell. 2011;146(6):873-887.

2. Carmeliet P, Jain RK. Principles and mechanisms of vessel normalization for cancer and other angiogenic disease. Nat Rev Drug Discov. 2011;10(6):417-427.

3. Apte RS, et al. VEGF in signaling and disease: beyond discovery and development. Cell. 2019;176(6):1248-1264.

4. Ferrara N, Adamis AP. Ten years of anti-vascular endothelial growth factor therapy. Nat Rev Drug Discov. 2016;15(6):385-403.

5. Ferrara N. Pathways mediating VEGF-independent tumor angiogenesis. Cytokine Growth Factor Rev. 2010;21(1):21-26.

6. Bergers G, Hanahan D. Modes of resistance to anti-angiogenic therapy. Nat Rev Cancer. 2008;8(8):592-603.

7. Weis SM, Cheresh DA. Tumor angiogenesis: molecular pathways and therapeutic targets. Nat Med. 2011;17(11):1359-1370.

8. Ebos JM, Kerbel RS. Antiangiogenic therapy: impact on invasion, disease progression, and metastasis. Nat Rev Clin Oncol. 2011;8(4):210-221.

9. Ratner M. Genentech discloses safety concerns over Avastin. Nat Biotechnol. 2004;22(10):1198.

10. Kamba T, McDonald DM. Mechanisms of adverse effects of anti-VEGF therapy for cancer. Br J Cancer. 2007;96(12):1788-1795.

11. Tian L, et al. Mutual regulation of tumour vesse normalization and immunostimulatory reprogramming. Nature. 2017;544(7649):250-254.

12. Jung $\mathrm{K}$, et al. Ly6Clo monocytes drive immunosuppression and confer resistance to anti-VEGFR2 cancer therapy. J Clin Invest. 2017;127(8):3039-3051.

13. Lacy SE, et al. Identification of FLRT1, FLRT2, and FLRT3: a novel family of transmembrane leucine-rich repeat proteins. Genomics. 1999;62(3):417-426.

14. Yamagishi S, et al. FLRT2 and FLRT3 act as repulsive guidance cues for Unc5-positive neurons. EMBO J. 2011;30(14):2920-2933.

15. Seiradake E, et al. FLRT structure: balancing repulsion and cell adhesion in cortical and vascular development. Neuron. 2014;84(2):370-385.

16. Tai-Nagara I, et al. Placental labyrinth formation in mice requires endothelial FLRT2/UNC5B signaling. Development. 2017;144(13):2392-2401.

17. Del Toro D, et al. Structural basis of teneurin-latrophilin interaction in repulsive guidance of migrating neurons. Cell. 2020;180(2):323-339.

18. Giannotta M, et al. VE-cadherin and endothelial adherens junctions: active guardians of vascular integrity. Dev Cell. 2013;26(5):441-454.

19. Weis S, et al. Endothelial barrier disruption by VEGF-mediated Src activity potentiates tumor cell extravasation and metastasis. J Cell Biol. 2004;167(2):223-229.
20. Myatt L, Cui X. Oxidative stress in the placenta. Histochem Cell Biol. 2004;122(4):369-382.

21. Okuno Y, et al. Pathological neoangiogenesis depends on oxidative stress regulation by ATM. Nat Med. 2012;18(8):1208-1216.

22. Jauhiainen S, et al. Axon guidance-related factor FLRT3 regulates VEGF-signaling and endothelial cell function. Front Physiol. 2019;10:224.

23. Müller PS, et al. The fibronectin leucine-rich repeat transmembrane protein Flrt2 is required in the epicardium to promote heart morphogenesis. Development. 2011;138(7):1297-1308.

24. Nowak-Sliwinska P, et al. Consensus guidelines for the use and interpretation of angiogenesis assays. Angiogenesis. 2018;21(3):425-532.

25. Armulik A, et al. Pericytes: developmental, physiological, and pathological perspectives, problems, and promises. Dev Cell. 2011;21(2):193-215.

26. Draoui N, et al. Angiogenesis revisited from a metabolic perspective: role and therapeutic implications of endothelial cell metabolism. Open Biol. 2017;7(12):170219.

27. Jain RK. Antiangiogenesis strategies revisited: from starving tumors to alleviating hypoxia. Cancer Cell. 2014;26(5):605-622.

28. Cantelmo AR, et al. Inhibition of the glycolytic activator PFKFB3 in endothelium induces tumor vesse normalization, impairs metastasis, and improves chemotherapy. Cancer Cell. 2016;30(6):968-985.

29. Park JS, et al. Normalization of tumor vessels by tie 2 activation and ang2 inhibition enhances drug delivery and produces a favorable tumor microenvironment. Cancer Cell. 2016;30(6):953-967.

30. Cairns RA, et al. Regulation of cancer cell metabolism. Nat Rev Cancer. 2011;11(2):85-95.

31. Bost F, et al. Energy disruptors: rising stars in anticancer therapy? Oncogenesis. 2016;18(1):e188

32. Kubo A, et al. Semi-quantitative analyses of metabolic systems of human colon cancer metastatic xenografts in livers of superimmunodeficient NOG mice. Anal Bioanal Chem. 2011;400(7):1895-1904.

33. Shimizu T, et al. c-MYC overexpression with loss of Ink $4 \mathrm{a} /$ Arf transforms bone marrow stromal cells into osteosarcoma accompanied by loss of adipogenesis. Oncogene. 2010;29(42):5687-5699.

34. Gengenbacher N, et al. Preclinical mouse solid tumour models: status quo, challenges and perspectives. Nat Rev Cancer. 2017;17(12):751-765.

35. Mazzone M, et al. Heterozygous deficiency of PHD2 restores tumor oxygenation and inhibits metastasis via endothelial normalization. Cell. 2009;136(5):839-851.

36. Su LK, et al. Multiple intestinal neoplasia caused by a mutation in the murine homolog of the APC gene. Science. 1992;256(5057):668-670.

37. Jackson VA, et al. Super-complexes of adhesion GPCRs and neural guidance receptors. Nat Commun. 2016;7:11184.
38. Camillo C, et al. LPHN2 inhibits vascular permeability by differential control of endothelial cell adhesion. JCell Biol. 2021;220(11):e202006033.

39. Sun Y, et al. Phosphorylation of caveolin-1 regulates oxidant-induced pulmonary vascular permeability via paracellular and transcellular pathways. Circ Res. 2009;105(7):676-685

40. Bae H, et al. Epigenetically regulated Fibronectin leucine rich transmembrane protein 2 (FLRT2) shows tumor suppressor activity in breast cancer cells. Sci Rep. 2017;7(1):272.

41. $\mathrm{Lu} \mathrm{X}$, et al. The netrin receptor UNC5B mediates guidance events controlling morphogenesis of the vascular system. Nature. 2004;432(7014):179-186.

42. Larrivée B, et al. Activation of the UNC5B receptor by Netrin-1 inhibits sprouting angiogenesis. Genes Dev. 2007;21(19):2433-2447.

43. Wilson BD, et al. Netrins promote developmental and therapeutic angiogenesis. Science. 2006;313(5787):640-644.

44. Castets $\mathrm{M}$, et al. Inhibition of endothelial cell apoptosis by netrin-1 during angiogenesis. Dev Cell. 2009;16(4):614-620.

45. Kobe B, Kajava AV. The leucine-rich repeat as a protein recognition motif. Curr Opin Struct Biol. 2001;11(6):725-732.

46. Xu Y, et al. FLRT2 promotes cellular proliferation and inhibits cell adhesion during chondrogenesis. J Cell Biochem. 2011;112(11):3440-3448.

47. Wei K, et al. Mouse FLRT2 interacts with the extracellular and intracellular regions of FGFR2. J Dent Res. 2011;90(10):1234-1239.

48. Okabe K, et al. Neurons limit angiogenesis by titrating VEGF in retina. Cell. 2014;159(3):584-596.

49. Hooper AT, et al. Engraftment and reconstitution of hematopoiesis is dependent on VEGFR2-mediated regeneration of sinusoidal endothelial cells. Cell Stem Cell. 2009;4(3):263-274.

50. Kawamoto S, et al. A novel reporter mouse strain that expresses enhanced green fluorescent protein upon Cre-mediated recombination. FEBS Lett. 2000;470(3):263-268

51 . Kubota Y, et al. Isolation and function of mouse tissue resident vascular precursors marked by myelin protein zero. JExp Med. 2011;208(5):949-960.

52. Sugiura $Y$, et al. Visualization and quantification of cerebral metabolic fluxes of glucose in awake mice. Proteomics. 2014;14(7-8):829-838.

53. Hu S, et al. Targeted metabolomic analysis of head and neck cancer cells using high performance ion chromatography coupled with a $\mathrm{Q}$ exactive HF mass spectrometer. Anal Chem. 2015;16(12):6371-6379.

54. Sugiura Y, et al. Visualization of in vivo metabolic flows reveals accelerated utilization of glucose and lactate in penumbra of ischemic heart. $\mathrm{Sci}$ Rep. 2016;1:32361. 